\title{
Integrated and Consistent Active Control Formulation and Piezotransducer Position Optimization of Plate Structures considering Spillover Effects
}

\author{
Mojtaba Biglar and Hamid Reza Mirdamadi \\ Department of Mechanical Engineering, Isfahan University of Technology, Isfahan 84156-83111, Iran \\ Correspondence should be addressed to Hamid Reza Mirdamadi; hrmirdamadi@cc.iut.ac.ir
}

Received 15 February 2014; Accepted 8 April 2014; Published 5 May 2014

Academic Editor: Chao Tao

Copyright (C) 2014 M. Biglar and H. R. Mirdamadi. This is an open access article distributed under the Creative Commons Attribution License, which permits unrestricted use, distribution, and reproduction in any medium, provided the original work is properly cited.

\begin{abstract}
This study addresses new formulation for active vibration control of plates by optimal locations of attached piezotransducers. Free vibrations are solved by Rayleigh-Ritz and transient by assumed modes methods. Optimal orientations of patches are determined by spatial controllability/observability, as well as residual modes to reduce spillover. These criteria are used to achieve optimal fitness function defined for genetic algorithm to find optimal locations. To control vibrations, negative velocity feedback control is designed. Results indicate that, by locating piezopatches at optimal positions, depreciation rate increases and amplitudes of vibrations reduce effectively. The effect of number of piezodevices is analyzed.
\end{abstract}

\section{Introduction}

In recent years, the active vibration control has been an important challenge for space structures. The importance has been more remarkable and higher for larger and more flexible space structures. For vibration suppression, smart materials such as piezoelectric transducers may be used $[1,2]$. Therefore, determining smart actuator and sensor locations is a key subject for increasing the system efficiency.

Many control algorithms have been used to eliminate the vibrations, such as positive position feedback (PPF) control and direct velocity feedback control [3,4]. Cupiał [5] proposed an optimization problem for finding the control voltage applied to a single piezoelectric patch actuator or several actuators for suppressing vibrations of a beam or rectangular plate. Yiqi and Yiming [6] established an analytical model for the active vibration control of a piezoelectric FGM plate based on a higher-order shear deformation plate and elastic piezoelectric theories. He et al. [7] presented a finite element formulation for the vibration control of a functionally graded plate (FGP) based on the classical lamination plate theory (CLPT). In the previous works of $[6,7]$, the plates were fully covered with integrated piezoelectric sensors and actuators and they employed piezoelectric patches. Thus, in these works, the optimization procedure was not required for an active vibration control. Orszulik and Shan [8] presented a system identification and vibration control strategy for a flexible manipulator with a collocated piezoelectric sensor/actuator pair. However, they did not perform any optimization on the location of piezoelectric transducers. Julai and Tokhi [9] presented an active vibration control mechanism using GA and particle swarm optimization. However, the optimization procedure for sensor/actuator locations was not performed in their work. Kulkarni et al. [10] presented numerical and experimental studies on active control of a coupled bending-torsion vibration modes of a typical wing box structure of an aircraft using piezoelectric ceramic stack actuators. Zhao and $\mathrm{Hu}$ [11] developed a simplified method for analyzing and designing against alleviating the effect of buffeting in vertical tail system. In [10, 11], an optimization procedure was not performed and the formulation would be different from that formulated in the present study.

Kumar and Narayanan [12] determined the optimal locations of the sensor/actuator pair on a flexible beam. In this work, they used the linear quadratic regulator (LQR) strategy for active vibration control. Bruant et al. [13] proposed a 
method based on minimizing the mechanical energy integral of the system for obtaining the optimal actuator locations and maximizing the energy of the state outputs for obtaining the optimal sensor locations on the flexible beam. Hać and Liu [14] derived analytical modeling of a smart plate equipped with piezoelectric sensors and actuators. In their work, two criteria for the optimal configuration of piezoelectric actuators were suggested using modal controllability and the controllability grammian.

Bruant et al. [15] developed a simple tool to simulate the active control of laminated plates. In a next step, they optimized the geometry and the number of sensors and actuators. Halim and Moheimani [16] suggested a criterion for finding the optimal placement of collocated piezoelectric actuator/sensor pairs on a thin flexible plate. However, in this work, not only the equations of motion were not of derived analytically but also the control algorithm had not been used for suppressing the plate vibration. Yang and Lee [17] developed an integer-real-encoded GA to search for the optimal placement and size of the piezoelectric patches. Rader et al. [18] presented an approach to optimizing the configuration of piezoelectric actuators for vibration control of a flexible aircraft fin. However, not only they derived the equations of motion of smart structure explicitly, but also the residual modes were not considered in their work. Ambrosio et al. [19] presented an $\mathrm{H}_{2}$ norm approach for the actuator and sensor placement. Sajizadeh and Darus [20] determined the optimal locations of sensors based on observability concept. Qian et al. [21] formulated the optimal actuator/sensor location problem in a framework of a zeroone optimization problem, which could be solved using GA. However, in this work, residual modes were neglected. Thus, spillover effects could distort the system performance. Bruant et al. [22] formulated the optimal locations and number of piezoelectric sensors for active vibration control. However, in $[20,22]$, they did not find the optimal locations of actuators.

The spillover effect could be among one of the most challenging issues that might spoil the active vibration control of structure. Indeed, when feedback controllers would be used for active vibration control of systems, there would be no assurance that control algorithm could excite the residual modes. Thus, for enhancing control algorithm operation, a consideration of residual modes should be necessary. However, in [14, 23, 24], residual modes were neglected. Thus, spillover effects could distort the system operation. Bruant et al. [25] and Han and Lee [26] determined the sensor and actuator locations with the consideration of controllability, observability, and spillover reduction. Furthermore, they used GA to find efficient locations of piezoelectric sensors/actuators. However, in their papers, the dynamic characteristics of both rectangular plate and piezoelectric sensors/actuators were not derived explicitly. Bruant and Proslier [27] presented an optimization problem based on optimization criteria of ensuring either good observability or good controllability of the structure, as well as considering residual modes to limit the spillover effects. However, in [27], the dynamic characteristics of both rectangular plate and piezoelectric sensors/actuators were not derived explicitly.

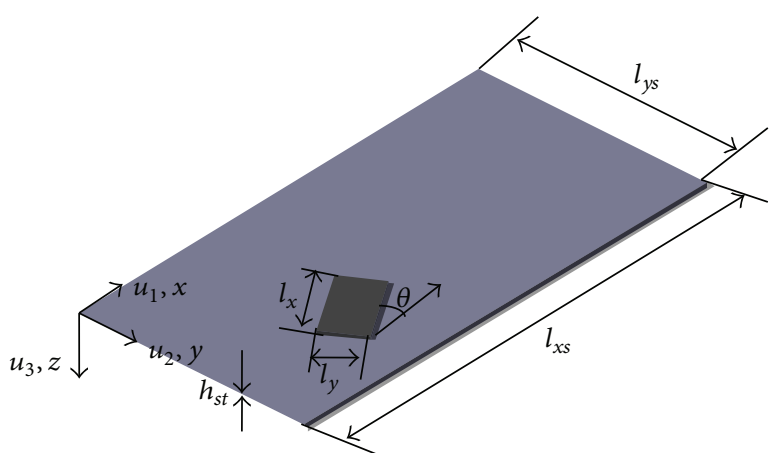

FIGURE 1: Flexible plate and orientation of piezoelectric patches.

In the present paper, an integrated and consistent formulation is derived based on energy principles and variational methods for both the host plate structure and the patch transducers, together with a GA optimization algorithm for optimal locations and orientations of these patches using spatial controllability-/observability-based optimality criteria. In addition, in this study, the effects of residual modes are incorporated in the fitness function of optimization algorithm. Based on the CPT and linear piezoelectric theory, the equations of motion and sensor output equations of the smart plate are derived by using Hamilton's principle, Rayleigh-Ritz approximation procedure, and the assumed modes method. For increasing the system efficiency, the optimal locations and orientations of piezoelectric transducers are determined based on spatial controllability and observability and considering residual modes to reduce the spillover effect. For active vibration control, a negative velocity feedback control algorithm is used.

The remainder of the paper is organized as follows. We derive the basic equation in Section 2. In Section 3, we discuss the optimization of the location of sensors and actuators mounted on a rectangular plate. The GA is used to find the optimal location of sensors and actuators in Section 4. In Section 5, designing controller for active vibration of plate and several simulations for showing the influence of optimization and active control algorithm are outlined. Finally, in Section 6, we draw conclusions.

\section{Basic Equations}

2.1. Potential and Kinetic Energies and Virtual Work. Consider a flexible plate (Figure 1) with $N_{a}$ piezoelectric actuators and $N_{\text {se }}$ piezoelectric sensors. The following formulation could be used for any plate geometry and any boundary conditions. The total potential energy of the structure and piezoelectric patch is expressed as [28]

$$
\begin{aligned}
\mathrm{PE}= & \int_{V^{\mathrm{st}}} \frac{1}{2} \mathbf{S}^{\mathrm{T}} \mathbf{c}_{\mathbf{s t}} \mathbf{S} \mathrm{d} V^{\mathrm{st}} \\
& +\sum_{j=1}^{N_{a}} \int_{V_{j}^{a}}\left(\frac{1}{2} \widetilde{\mathbf{S}}^{\mathrm{T}} \mathbf{c}^{\mathbf{D}} \widetilde{\mathbf{S}}-\widetilde{\mathbf{S}}^{\mathrm{T}} \mathbf{h} \widetilde{\mathbf{D}}^{\mathbf{j}}+\frac{1}{2} \widetilde{\mathbf{D}}^{\mathrm{jT}} \boldsymbol{\beta}_{\mathbf{S}} \widetilde{\mathbf{D}}^{\mathbf{j}}\right) \mathrm{d} V_{j}^{a} \\
& +\sum_{j=1}^{N_{\mathrm{se}}} \int_{V_{j}^{\mathrm{se}}}\left(\frac{1}{2} \widetilde{\mathbf{S}}^{\mathrm{T}} \mathbf{c}^{\mathbf{D}} \widetilde{\mathbf{S}}-\widetilde{\mathbf{S}}^{\mathrm{T}} \mathbf{h} \widetilde{\mathbf{D}}^{\mathbf{j}}+\frac{1}{2} \widetilde{\mathbf{D}}^{\mathbf{j T}} \boldsymbol{\beta}_{\mathbf{S}} \widetilde{\mathbf{D}}^{\mathbf{j}}\right) \mathrm{d} V_{j}^{\mathrm{se}},
\end{aligned}
$$


where $\widetilde{\mathbf{S}}$ and $\widetilde{\mathbf{D}}^{\mathfrak{j}}$ are strain and electric charge density vector in local coordinates, respectively. $\mathbf{c}_{\mathrm{st}}, \mathrm{c}^{\mathrm{D}}, \mathbf{h}$, and $\boldsymbol{\beta}_{\mathrm{S}}$ are the matrices of elastic constants of structure, elastic constants of piezoelectric patches under constant electric charge density, the piezoelectric constants, and the inverse of dielectric constant under constant strain; $\widetilde{\mathbf{S}}^{\mathrm{T}}$ and $\widetilde{\mathbf{D}}^{\mathrm{jT}}$ are the transposes of $\widetilde{\mathbf{S}}$ and $\widetilde{\mathbf{D}}^{\mathbf{j}} . V^{\text {st }}, V^{a}$, and $V^{\text {se }}$ are the volumes of structure, piezoelectric actuators, and piezoelectric sensors, respectively. $N_{a}$ and $N_{\text {se }}$ are the number of actuator and sensor patches. By using the following relation, the local coordinate of system for each piezoelectric element can be transformed to global coordinates:

$$
\widetilde{\mathbf{S}}=\mathbf{R}_{\mathbf{S}}^{\mathbf{j}} \mathbf{S}, \quad \widetilde{\mathbf{D}}^{\mathbf{j}}=\mathbf{R}_{\mathrm{D}}^{\mathbf{j}} \mathbf{D}^{\mathbf{j}},
$$

where $\mathbf{R}_{\mathbf{S}}^{\mathbf{j}}$ and $\mathbf{R}_{\mathbf{D}}^{\mathbf{j}}$ are strain and electric charge density transformation matrices, respectively. The total kinetic energy of the structure and piezoelectric patches are obtained as [28]

$$
\begin{aligned}
\mathrm{KE}= & \frac{1}{2} \int_{V^{\mathrm{st}}} \rho^{\mathrm{st}}\left(\dot{u}_{1}^{2}+\dot{u}_{2}^{2}+\dot{u}_{3}^{2}\right) \mathrm{d} V_{\mathrm{st}} \\
& +\frac{1}{2} \sum_{j=1}^{N_{a}} \int_{V_{j}^{a}} \rho_{j}^{a}\left(\dot{u}_{1}^{2}+\dot{u}_{2}^{2}+\dot{u}_{3}^{2}\right) \mathrm{d} V_{j}^{a} \\
& +\frac{1}{2} \sum_{j=1}^{N_{\mathrm{se}}} \int_{V_{j}^{\mathrm{se}}} \rho_{j}^{\mathrm{se}}\left(\dot{u}_{1}^{2}+\dot{u}_{2}^{2}+\dot{u}_{3}^{2}\right) \mathrm{d} V_{j}^{\mathrm{se}} \\
= & \frac{1}{2} \int_{V^{\mathrm{st}}} \rho^{\mathrm{st}}\left(\dot{\mathbf{u}}^{\mathrm{T}} \dot{\mathbf{u}}\right) \mathrm{d} V_{\mathrm{st}}+\frac{1}{2} \sum_{j=1}^{N_{a}} \int_{V_{j}^{a}} \rho_{j}^{a}\left(\dot{\mathbf{u}}^{\mathrm{T}} \dot{\mathbf{u}}\right) \mathrm{d} V_{j}^{a} \\
& +\frac{1}{2} \sum_{j=1}^{N_{\mathrm{se}}} \int_{V_{j}^{\mathrm{se}}} \rho_{j}^{\mathrm{se}}\left(\dot{\mathbf{u}}^{\mathrm{T}} \dot{\mathbf{u}}\right) \mathrm{d} V_{j}^{\mathrm{se}},
\end{aligned}
$$

where $\dot{u}_{1}, \dot{u}_{2}$, and $\dot{u}_{3}$ are the velocity components in the $x, y$, and $z$ directions and $\rho^{\text {st }}, \rho^{a}$, and $\rho^{\text {se }}$ are the mass densities of structure, piezoelectric actuators, and piezoelectric sensors, respectively. The virtual work of external forces is written as [28]

$$
\delta W^{\mathrm{ext}}=\sum_{j=1}^{N_{a}} v_{j}^{a} \delta q_{j}^{a}+\sum_{j=1}^{N_{\mathrm{se}}} v_{j}^{\mathrm{se}} \delta q_{j}^{\mathrm{se}}+\sum_{j=1}^{N_{f}} \delta \mathbf{u}\left(x_{j}, y_{j}\right)^{T} f_{j},
$$

where $v$ and $f$ are voltage that applied to piezoelectric patches and concentrated force and $\delta q$ and $\delta \mathbf{u}$ are variation of electric charge and mechanical displacement.

2.2. Discretized Displacement Field. In the Rayleigh-Ritz method, the spatial displacement field of midplane for a vibrating rectangular plate is written as

$$
\begin{aligned}
& u_{1}(x, y, t)=\mathbf{N}_{\mathbf{u}_{1}}(x, y) \mathbf{U}_{1}(t), \\
& u_{2}(x, y, t)=\mathbf{N}_{\mathbf{u}_{2}}(x, y) \mathbf{U}_{2}(t), \\
& u_{3}(x, y, t)=\mathbf{N}_{\mathbf{u}_{3}}(x, y) \mathbf{U}_{3}(t),
\end{aligned}
$$

where $u_{1}, u_{2}$, and $u_{3}$ are the midplane displacements of the plate along the $x, y$, and $z$ directions, respectively. $\mathbf{U}_{1}, \mathbf{U}_{2}$, and $\mathbf{U}_{3}$ are the generalized coordinates of plate response, and $\mathbf{N}_{\mathbf{u}_{1}}, \mathbf{N}_{\mathbf{u}_{2}}$, and $\mathbf{N}_{\mathbf{u}_{3}}$ are the shape function that need to satisfy geometric boundary conditions. Equation (5) can be expressed as a matrix relationship:

$$
\mathbf{u}=\left\{\begin{array}{l}
u_{1} \\
u_{2} \\
u_{3}
\end{array}\right\}=\left[\begin{array}{ccc}
\mathbf{N}_{\mathbf{u}_{1}} & \mathbf{0} & \mathbf{0} \\
\mathbf{0} & \mathbf{N}_{\mathbf{u}_{2}} & \mathbf{0} \\
\mathbf{0} & \mathbf{0} & \mathbf{N}_{\mathbf{u}_{3}}
\end{array}\right]\left\{\begin{array}{l}
\mathbf{U}_{1} \\
\mathbf{U}_{2} \\
\mathbf{U}_{3}
\end{array}\right\}=\mathbf{N}_{\mathbf{u}} \mathbf{U} .
$$

2.3. Discretized Strain-Displacement Relationship. The relationship between strain and displacement, based on the classical plate theory, is expressed as [28]

$$
\left\{\begin{array}{l}
S_{x x} \\
S_{y y} \\
S_{x y}
\end{array}\right\}=\left\{\begin{array}{c}
\frac{\partial u_{1}}{\partial x} \\
\frac{\partial u_{2}}{\partial y} \\
\frac{\partial u_{1}}{\partial y}+\frac{\partial u_{2}}{\partial x}
\end{array}\right\}+z\left\{\begin{array}{c}
-\frac{\partial^{2} u_{3}}{\partial x^{2}} \\
-\frac{\partial u_{3}}{\partial x} \\
-2 \frac{\partial^{2} u_{3}}{\partial x \partial y}
\end{array}\right\}
$$

In terms of generalized coordinates, (7) becomes

$$
\begin{aligned}
\left\{\begin{array}{l}
S_{x x} \\
S_{y y} \\
S_{x y}
\end{array}\right\}= & {\left[\begin{array}{ccc}
\frac{\partial \mathbf{N}_{\mathbf{u}_{1}}}{\partial x} & \mathbf{0} & \mathbf{0} \\
\mathbf{0} & \frac{\partial \mathbf{N}_{\mathbf{u}_{2}}}{\partial y} & \mathbf{0} \\
\frac{\partial \mathbf{N}_{\mathbf{u}_{1}}}{\partial y} & \frac{\partial \mathbf{N}_{\mathbf{u}_{2}}}{\partial x} & \mathbf{0}
\end{array}\right]\left\{\begin{array}{l}
\mathbf{U}_{\mathbf{1}} \\
\mathbf{U}_{2} \\
\mathbf{U}_{3}
\end{array}\right\} } \\
& +\left[\begin{array}{ccc}
\mathbf{0} & \mathbf{0} & -z \frac{\partial^{2} \mathbf{N}_{\mathbf{u}_{3}}}{\partial x^{2}} \\
\mathbf{0} & \mathbf{0} & -z \frac{\partial^{2} \mathbf{N}_{\mathbf{u}_{3}}}{\partial y^{2}} \\
\mathbf{0} & \mathbf{0} & -z \frac{\partial^{2} \mathbf{N}_{\mathbf{u}_{3}}}{\partial x \partial y}
\end{array}\right]\left\{\begin{array}{l}
\mathbf{U}_{1} \\
\mathbf{U}_{2} \\
\mathbf{U}_{3}
\end{array}\right\} \\
= & \mathbf{B}_{\mathbf{U}}^{\mathbf{m}} \mathbf{U}+\mathbf{B}_{\mathbf{U}}^{\mathbf{b}} \mathbf{U}
\end{aligned}
$$

where $\mathbf{B}_{\mathrm{U}}^{\mathbf{m}}$ and $\mathbf{B}_{\mathrm{U}}^{\mathbf{b}}$ are, respectively, strain-displacement transformation matrices for membrane and flexural actions.

2.4. Discretized Electric Charge Density. In this subsection, we attempt to discrete the electric charge density. For achieving this purpose, we assume that the electric charge density is constant within a layer of a patch. Additionally, we assume that electric charge density acts only in the thickness direction of piezoelectric patches and the electric charge on each patch is chosen as a generalized coordinate. With this assumption, a mathematical relationship between electric 
charge density and the corresponding generalized coordinate for each piezoelectric patch $j$ is written as [28]

$$
\begin{gathered}
\mathbf{D}^{j}=\mathbf{B}_{\mathbf{q}}^{j} \mathbf{q}^{p} \Longrightarrow \mathbf{B}_{\mathbf{q}}^{1}=\left[\begin{array}{cccc}
0 & 0 & & 0 \\
0 & 0 & \cdots & 0 \\
\frac{1}{A_{p}^{1}} & 0 & & 0
\end{array}\right], \\
\mathbf{B}_{\mathbf{q}}^{2}=\left[\begin{array}{llll}
0 & 0 & & 0 \\
0 & 0 & \cdots & 0 \\
0 & \frac{1}{A_{p}^{2}} & & 0
\end{array}\right], \quad \mathbf{B}_{\mathbf{q}}^{N^{p}}=\left[\begin{array}{llll}
0 & 0 & & 0 \\
0 & 0 & \cdots & 0 \\
0 & 0 & & \frac{1}{A_{p}^{N^{p}}}
\end{array}\right],
\end{gathered}
$$

where $A$ is the area of piezoelectric patches. By using (9), the electrical generalized coordinate vector is defined as

$$
\mathbf{q}^{p}=\left[\begin{array}{c}
q_{1}^{p} \\
q_{2}^{p} \\
q_{3}^{p} \\
\vdots \\
q_{N_{p}}^{p}
\end{array}\right],
$$

where $p=a$ or $p=$ se. In this content, a means actuator and se means sensor. Inserting (8) and (9) into the equation of potential energy, that is, (1), yields the following expression that is a function of generalized coordinates [28]:

$$
\begin{aligned}
\mathrm{PE}= & \frac{1}{2} \mathbf{U}^{\mathrm{T}} \mathbf{K}^{\mathrm{st}} \mathbf{U}+\frac{1}{2} \mathbf{U}^{\mathrm{T}} \mathbf{K}_{\mathrm{D}}^{\mathrm{se}} \mathbf{U}+\frac{1}{2} \mathbf{U}^{\mathrm{T}} \mathbf{K}_{\mathrm{D}}^{\mathrm{a}} \mathbf{U} \\
& -\mathbf{U}^{\mathrm{T}} \mathbf{K}_{\mathrm{Uq}}^{\mathrm{se}} \mathbf{q}^{\mathrm{se}}-\mathbf{U}^{\mathrm{T}} \mathbf{K}_{\mathrm{Uq}}^{\mathrm{a}} \mathbf{q}^{\mathrm{a}} \\
& +\frac{1}{2} \mathbf{q}^{\text {seT }} \mathbf{K}_{\mathbf{q q}}^{\mathrm{se}} \mathbf{q}^{\mathrm{se}}+\frac{1}{2} \mathbf{q}^{\mathrm{aT}} \mathbf{K}_{\mathbf{q q}}^{\mathrm{a}} \mathbf{q}^{\mathrm{a}},
\end{aligned}
$$

where $\mathbf{K}^{\text {st }}, \mathbf{K}_{\mathrm{D}}^{\text {se }}$, and $\mathbf{K}_{\mathbf{D}}^{\mathbf{a}}$ are, respectively, the stiffness matrices of the structure, piezoelectric sensors, and piezoelectric actuators, $\mathbf{K}_{\mathbf{U q}}^{\text {se }}$ and $\mathbf{K}_{\mathbf{U q}}^{\mathrm{a}}$ are coupling matrices of sensors and actuators, and $\mathbf{K}_{\mathbf{q q}}^{\text {se }}$ and $\mathbf{K}_{\mathbf{q q}}^{\mathrm{a}}$ are capacitance matrices of sensors and actuators, respectively. The matrices are calculated as

$$
\begin{aligned}
& K^{s t}=\int_{V^{s t}}\left(B_{U}^{m T} c_{s t} B_{U}^{m}+B_{U}^{b^{T}} c_{s t} B_{U}^{b}+B_{U}^{m^{T}} c_{s t} B_{U}^{b}\right. \\
& \left.+\mathbf{B}_{\mathbf{U}}^{\mathbf{b}^{\mathbf{T}}} \mathbf{c}_{\mathbf{s t}} \mathbf{B}_{\mathrm{U}}^{\mathbf{m}}\right) \mathrm{d} V^{\mathrm{st}}
\end{aligned}
$$

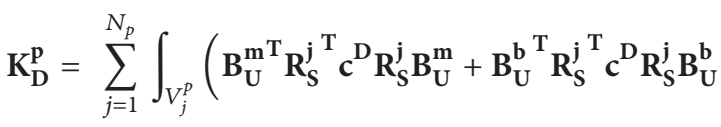

$$
\begin{aligned}
& \left.+B_{U}^{m^{T}} R_{S}^{j}{ }^{\mathrm{T}} c^{\mathrm{D}} R_{S}^{\mathrm{j}} B_{U}^{b}+B_{U}^{b^{\mathrm{T}}} \mathbf{R}_{S}^{\mathrm{j}}{ }^{\mathrm{T}} c^{\mathrm{D}} R_{S}^{\mathrm{j}} B_{U}^{\mathrm{m}}\right) \\
& \times \mathrm{d} V_{j}^{p}, \\
& \mathbf{K}_{\mathbf{U} \mathbf{q}}^{p}=\sum_{j=1}^{N_{p}}\left(\int_{V_{j}^{p}} \mathbf{B}_{\mathbf{U}}^{\mathbf{m}^{\mathrm{T}}} \mathbf{R}_{\mathbf{S}}^{\mathbf{j}}{ }^{\mathrm{T}} \mathbf{h} \mathbf{R}_{\mathbf{D}}^{\mathbf{j}} \mathbf{B}_{\mathbf{q}}^{\mathbf{j}} \mathrm{d} V_{j}^{p}\right. \\
& \left.+\int_{V_{j}^{p}} \mathbf{B}_{\mathbf{U}}^{\mathbf{b}^{\mathrm{T}}} \mathbf{R}_{\mathbf{S}}^{\mathbf{j}^{\mathrm{T}}} \mathbf{h R}_{\mathbf{D}}^{\mathbf{j}} \mathbf{B}_{\mathbf{q}}^{\mathbf{j}} \mathrm{d} V_{j}^{p}\right), \\
& \mathbf{K}_{\mathbf{q} \mathbf{q}}^{\mathbf{p}}=\sum_{j=1}^{N_{p}} \int_{V_{j}^{p}} \mathbf{B}_{\mathbf{q}}^{\mathbf{j}}{ }^{\mathrm{T}} \mathbf{R}_{\mathbf{D}}^{\mathbf{j}}{ }^{\mathbf{T}} \boldsymbol{\beta}_{\mathbf{s}} \mathbf{R}_{\mathbf{D}}^{\mathbf{j}} \mathbf{B}_{\mathbf{q}}^{\mathbf{j}} \mathrm{d} V_{j}^{p},
\end{aligned}
$$

where $p=a$ or $p=$ se. If we insert (6) into (3), kinetic energy can be rewritten as

$$
\mathrm{KE}=\frac{1}{2} \dot{\mathbf{U}} \mathbf{M}^{\mathrm{st}} \dot{\mathrm{U}}+\frac{1}{2} \dot{\mathbf{U}} \mathbf{M}^{\mathrm{se}} \dot{\mathbf{U}}+\frac{1}{2} \dot{\mathbf{U}} \mathbf{M}^{\mathrm{a}} \dot{\mathbf{U}},
$$

where $\mathbf{M}^{\text {st }}, \mathbf{M}^{\text {se }}$, and $\mathbf{M}^{\mathbf{a}}$ are mass matrices of the plate, piezoelectric sensors, and piezoelectric actuators. The matrices are calculated as

$$
\begin{aligned}
& \mathbf{M}^{\mathrm{st}}=\int_{V^{\mathrm{st}}} \rho^{\mathrm{st}} \mathbf{N}_{\mathbf{u}}^{\mathrm{T}} \mathbf{N}_{\mathbf{u}} \mathrm{d} V^{\mathrm{st}}, \\
& \mathbf{M}^{\mathbf{p}}=\sum_{j=1}^{N_{p}} \int_{V_{j}^{p}} \rho_{j}^{p} \mathbf{N}_{\mathbf{u}}^{\mathrm{T}} \mathbf{N}_{\mathbf{u}} \mathrm{d} V_{j}^{p},
\end{aligned}
$$

where $p=a$ or $p=$ se.

By inserting (6) into (4), the external work can be written as

$$
\delta W^{\mathrm{ext}}=\delta \mathbf{q}^{\mathrm{a} \mathrm{T}} \mathbf{B}_{\mathbf{V}}^{\mathrm{a}} \mathbf{V}^{\mathrm{a}}+\delta \mathbf{q}^{\mathrm{seT}} \mathbf{B}_{\mathrm{V}}^{\mathrm{se}} \mathbf{V}^{\mathrm{se}}+\delta \mathbf{U}^{\mathrm{T}} \mathbf{F}_{\mathbf{c}},
$$

where $\mathbf{F}_{\mathbf{c}}$ is

$$
\mathbf{F}_{\mathbf{c}}=\sum_{j=1}^{N_{f}} \mathbf{N}_{\mathbf{u}}^{\mathrm{T}}\left(x_{j}, y_{j}\right) f_{j} .
$$

In addition, $\mathbf{B}_{\mathrm{V}}^{\mathbf{a}}$ and $\mathbf{B}_{\mathrm{V}}^{\text {se }}$ are defined as

$$
\mathbf{B}_{\mathrm{v}}^{\mathrm{a}}=\mathbf{I}_{\mathrm{N}_{\mathrm{a}}}, \quad \mathbf{B}_{\mathrm{v}}^{\mathrm{se}}=\mathbf{I}_{\mathrm{N}_{\mathrm{se}}},
$$

where $\mathbf{I}$ is an identity matrix. 
2.5. Governing Equations of Motion. Utilizing Hamilton's principle, the governing equations for the plate vibration equipped with piezoelectric sensor and actuator patches can be derived:

$$
\begin{gathered}
\mathbf{M U ̈}+\mathbf{K}_{\mathbf{U U}} \mathbf{U}-\mathbf{K}_{\mathbf{U q}}^{\mathrm{se}} \mathbf{q}^{\mathrm{se}}-\mathbf{K}_{\mathbf{U q}}^{\mathbf{a}} \mathbf{q}^{\mathbf{a}}=\mathbf{F}, \\
-\mathbf{K}_{\mathbf{q U}}^{\mathrm{se}} \mathbf{U}+\mathbf{K}_{\mathbf{q q}}^{\mathrm{se}} \mathbf{q}^{\mathrm{se}}=\mathbf{B}_{\mathbf{v}}^{\mathrm{se}} \mathbf{v}(t)^{\mathrm{se}}, \\
-\mathbf{K}_{\mathbf{q U}}^{\mathbf{a}} \mathbf{U}+\mathbf{K}_{\mathbf{q q}}^{\mathrm{a}} \mathbf{q}^{\mathbf{a}}=\mathbf{B}_{\mathbf{v}}^{\mathbf{a}} \mathbf{v}(t)^{\mathbf{a}},
\end{gathered}
$$

where $\mathbf{K}_{\mathbf{U q}}=\mathbf{K}_{\mathbf{q U}}{ }^{\mathrm{T}}, \mathbf{M}=\mathbf{M}^{\text {st }}+\mathbf{M}^{\text {se }}+\mathbf{M}^{\mathbf{a}}$, and $\mathbf{K}_{\mathbf{U U}}=$ $\mathbf{K}^{\text {st }}+\mathbf{K}^{\text {se }}+\mathbf{K}^{\mathbf{a}}$. Further, $\mathbf{v}(t)^{\mathbf{a}}$ and $\mathbf{v}(t)^{\text {se }}$ are the external voltages applied to the actuators and sensors. $\mathbf{M}$ and $\mathbf{K}_{\mathbf{U U}}$ are, respectively, the total mass and passive stiffness matrices of the system and (19) can be rewritten as

$$
\mathbf{q}^{\mathrm{se}}=\mathbf{K}_{\mathrm{qq}}^{\mathrm{se}-1} \mathbf{B}_{\mathbf{v}}^{\mathrm{se}} \mathbf{v}^{\mathrm{se}}+\mathbf{K}_{\mathrm{qq}}^{\mathrm{se}-1} \mathbf{K}_{\mathrm{qU}}^{\mathrm{se}} \mathbf{U} .
$$

It is supposed that there is no external applied voltage across the sensor patches; thus $\mathbf{v}^{\text {se }}=0$. Doing the above operation for (20) results in actuator electric charge:

$$
\mathbf{q}^{\mathrm{a}}=\mathrm{K}_{\mathrm{qq}}^{\mathrm{a}-1} \mathbf{B}_{\mathrm{v}}^{\mathrm{a}} \mathbf{v}^{\mathrm{a}}+\mathrm{K}_{\mathrm{qq}}^{\mathrm{a}-1} \mathrm{~K}_{\mathrm{qU}}^{\mathrm{a}} \mathrm{U}
$$

Substituting (21) and (22) into (18) yields the following expression for the equations of motion of the plate:

$$
\mathbf{M U ̈}+\mathbf{K U}=\Upsilon_{\mathbf{a}} \mathbf{v}^{\mathbf{a}}+\mathbf{F}_{\mathbf{c}},
$$

where

$$
\Upsilon_{\mathbf{a}}=\mathbf{K}_{\mathbf{U q}}^{\mathrm{a}} \mathbf{K}_{\mathbf{q q}}^{\mathrm{a}-1} \mathbf{B}_{\mathbf{v}}^{\mathrm{a}}
$$

is the influence matrix of input voltage applied across actuator patches. The total active and passive matrix of system stiffness is expressed as follows:

$$
\mathbf{K}=\mathbf{K}_{\mathrm{UU}}-\mathbf{K}_{\mathrm{Uq}}^{\mathrm{se}} \mathbf{K}_{\mathbf{q q}}^{\mathrm{se}-1} \mathbf{K}_{\mathbf{q U}}^{\mathrm{se}}-\mathbf{K}_{\mathrm{Uq}}^{\mathrm{a}} \mathbf{K}_{\mathbf{q q}}^{\mathrm{a}-1} \mathbf{K}_{\mathbf{q U}}^{\mathrm{a}} .
$$

The current through the thickness of each sensor patch is equal to the time derivative of the electric charge accumulated on the surface of each sensor as given in (21):

$$
\mathbf{i}^{\text {se }}(t)=\frac{\mathrm{dq}^{\text {se }}(t)}{\mathrm{d} t} \text {. }
$$

Whenever a piezoelectric sensor is used for sensing a strain rate, the current can be converted into the output open circuit sensor voltage [3]:

$$
\Phi^{\mathbf{s e}}(t)=\mathbf{G}_{\mathbf{c}} \mathbf{i}(t)
$$

where $\mathbf{G}_{\mathbf{c}}$ is the matrix of constant gains of the current amplifier and converts the sensor current vector to an output sensor voltage vector. Using (21) and (26), the output sensor voltage can be expressed as

$$
\Phi^{\mathrm{se}}(t)=\mathbf{G}_{\mathbf{c}} \mathbf{K}_{\mathbf{q q}}^{\mathrm{se}-1} \mathbf{K}_{\mathbf{q U}}^{\mathrm{se}} \dot{\mathbf{U}}=\mathbf{C} \dot{\mathbf{U}}
$$

where (28) is the sensor equation, which transforms the strain rate to a voltage.
2.6. Modal Equations. The eigenvalue problem of (23) can be solved for determining the eigenvalues and eigenvectors. The orthonormality property must be satisfied as

$$
\mathbf{H}^{\mathrm{T}} \mathbf{M H}=\mathbf{I}, \quad \mathbf{H}^{\mathrm{T}} \mathbf{K H}=\Lambda_{\mathbf{n}}^{2},
$$

where $n$ is the number of the modes, $\Lambda_{\mathbf{n}}^{2}=\operatorname{diag}\left(\left[\begin{array}{ll}\omega_{1}^{2} & \omega_{2}^{2}\end{array}\right.\right.$ $\left.\omega_{3}^{2} \quad \cdots \omega_{n}^{2}\right]$ ) is the eigenvalue matrix, and $\mathbf{H}$ is the eigenvector matrix. The modal coordinates are introduced as

$$
\mathbf{U}=\mathbf{H R} \text {. }
$$

$\mathbf{R}$ is generalized displacement vector. By using modal coordinates, modal equations of motion and modal sensor equation can be obtained as

$$
\ddot{\mathbf{R}}+\Lambda_{\mathbf{n}}^{2} \mathbf{R}=\Upsilon_{\mathbf{a}}^{\prime} \mathbf{v}^{\mathrm{a}}+\mathbf{H}^{\mathrm{T}} \mathbf{F}_{\mathrm{c}}, \quad \Phi^{\mathrm{se}}(\mathbf{t})=\mathbf{C}^{\prime} \dot{\mathbf{R}},
$$

where $\Upsilon_{\mathbf{a}}^{\prime}=\mathbf{H}^{\mathrm{T}} \Upsilon_{\mathbf{a}}$ and $\mathbf{C}^{\prime}=\mathbf{C H}$. The addition of a structural damping ratio matrix into the modal equations of motion results in the following expressions [29]:

$$
\ddot{\mathbf{R}}+2 \mathrm{Z} \Lambda_{\mathbf{n}} \dot{\mathbf{R}}+\Lambda_{\mathbf{n}}^{2} \mathbf{R}=\Upsilon_{\mathbf{a}}^{\prime} \mathbf{v}^{\mathrm{a}}+\mathbf{H}^{\mathrm{T}} \mathbf{F}_{\mathbf{c}},
$$

where $\mathbf{Z}$ is a diagonal matrix of modal damping ratios, $\Lambda_{\mathbf{n}}$ is a diagonal matrix of natural frequencies, and $\Lambda_{n}^{2}$ is a diagonal matrix which is equal to the square of natural frequencies. The matrices of $\mathbf{Z}, \Lambda_{\mathbf{n}}$, and $\Lambda_{\mathbf{n}}^{2}$ are written as

$$
\begin{aligned}
\mathbf{Z} & =\left[\begin{array}{ccccc}
\zeta_{1} & 0 & \cdots & 0 & 0 \\
0 & \zeta_{2} & & 0 & 0 \\
& \vdots & \ddots & \vdots & \\
0 & 0 & & \zeta_{n-1} & 0 \\
0 & 0 & \cdots & 0 & \zeta_{n}
\end{array}\right], \\
\Lambda_{\mathbf{n}} & =\left[\begin{array}{ccccc}
\omega_{1} & 0 & \cdots & 0 & 0 \\
0 & \omega_{2} & & 0 & 0 \\
& \vdots & \ddots & \vdots & \\
0 & 0 & & \omega_{n-1} & 0 \\
0 & 0 & \cdots & 0 & \omega_{n}
\end{array}\right] .
\end{aligned}
$$

\section{Optimal Locations of the Piezoelectric Sensors and Actuators}

To determine the optimal piezoelectric sensor and actuator locations for a simply supported plate, a modified optimization criterion based on the concept of spatial $\mathscr{H}_{2}$ norm is used [30]. In this method, two criteria for determining the optimal placement of piezoelectric actuators and sensors are proposed, using spatial controllability/observability and considering the effect of residual modes to reduce the spillover effect for the simply supported plate. Here, we consider a state of no in-plane extension and assume that the plate is only in a condition of pure bending. With this assumption, the spatially discredited displacement field of the midplane is written as

$$
u_{1}=0, \quad u_{2}=0, \quad u_{3}=\mathbf{N}_{\mathbf{u}_{3}} \mathbf{U}_{3} .
$$


3.1. Optimal Placement of Actuators. We assume the voltages applied to the actuator patches are as follows:

$$
\mathbf{v}^{\mathbf{a}}=\left[\begin{array}{llll}
v_{1}^{a} & v_{2}^{a} & \cdots & v_{N_{a}}^{a}
\end{array}\right]^{T}
$$

By the assumption that the initial conditions and external mechanical forces are zero, the transfer function of the plate, resulting from Laplace transforming of (32), can be written as

$$
G(s, x, y)=\sum_{i=1}^{n} G_{i}(s, x, y)=\sum_{i=1}^{n} \frac{N_{u_{3}}^{i} \Upsilon_{a}^{i}}{s^{2}+2 \zeta_{i} \omega_{i} s+\omega_{i}^{2}} .
$$

Equation (36) is the transfer function from the voltages applied to the actuator patches, $\mathbf{v}(t)^{\mathbf{a}}$, to the plate pure bending, $u_{3} . \Upsilon_{a}{ }^{i}$ is defined as

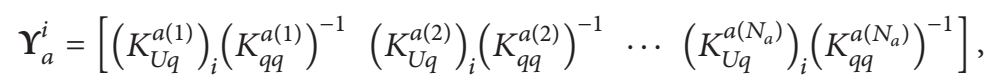

and $N_{u_{3}}^{i}$ is the $i$ th component of the vector $\mathbf{N}_{\mathbf{u}_{3}}$. Now, we are in a position to define the spatial $\mathscr{H}_{2}$ norm of a transfer function as $[28,31,32]$

$$
\begin{aligned}
\|G\|_{2}^{2}= & \int_{-\infty}^{+\infty} \int_{0}^{l_{x s}} \int_{0}^{l_{y s}} \operatorname{trace}\{G(j \omega, x, y) * G(j \omega, x, y)\} \\
& \times d y d x d \omega \\
= & \sum_{i=1}^{n}\left\|\widetilde{G}_{i}\right\|_{2}^{2},
\end{aligned}
$$

where trace is defined to be the sum of the elements on the main diagonal of a matrix and

$$
\widetilde{G}_{i}=\frac{\Upsilon_{a}^{i}}{s^{2}+2 \zeta_{i} \omega_{i} s+\omega_{i}^{2}}, \quad i=1, \ldots, n .
$$

We find the optimal location of the $j$ th piezoelectric actuator patch by defining the spatial controllability, function $\psi_{i j}$, as

$$
\begin{aligned}
\psi_{i j}\left(x^{a(j)}, y^{a(j)}, \theta^{a(j)}\right) \\
=\left\|\widetilde{G}_{i}^{j}\right\|_{2} \\
=\left|\left(K_{U q}^{a(j)}\right)_{i}\left(K_{q q}^{a(j)}\right)^{-1}\right|\left\|\frac{\Upsilon_{a}^{i}}{s^{2}+2 \zeta_{i} \omega_{i} s+\omega_{i}^{2}}\right\|, \\
i=1, \ldots, n,
\end{aligned}
$$

where $\left(x^{a(j)}, y^{a(j)}\right)$ and $\theta^{a(j)}$ are the corner coordinates of the $j$ th actuator patch and its orientation, respectively. The actuator orientation may change in the interval, $(0 \leq \theta<$ $\pi)$. In this study, the variables of the actuator locations that should be optimized are $\left\{\left(x^{a(j)}, y^{a(j)}, \theta^{a(j)}\right)\right\}, j=1,2, \ldots, N_{a}$. The patches orientation is shown in Figure 1.

For enhancing the performance of feedback control, we should reduce the spillover effects. For achieving this purpose, it is necessary that, with due consideration of the residual modes, we minimize the authority of the actuators over the high-frequency modes. Furthermore, we can enhance the performance of control with considering only the first $N_{C}$ modes and place the actuators at the locations that they provide a high-authority over the first $N_{C}$ modes.
The actuators optimal locations can be found by maximizing the following criterion:

$$
\begin{aligned}
& \sum_{j=1}^{N_{a}} \sqrt{\sum_{i=1}^{N_{C}} \psi_{i j}\left(x^{a(j)}, y^{a(j)}, \theta^{a(j)}\right)^{2}} \\
& \quad-\lambda \sum_{j=1}^{N_{a}} \sqrt{\sum_{i=N_{C}}^{N_{C}+N_{R}} \psi_{i j}\left(x^{a(j)}, y^{a(j)}, \theta^{a(j)}\right)^{2}},
\end{aligned}
$$

where $\lambda$ is a weighting constant. $N_{C}$ and $N_{R}$ are number of controlled and residual modes. The terms, $\sqrt{\sum_{i=1}^{N_{C}} \psi_{i j}\left(x^{a(j)}, y^{a(j)}, \theta^{a(j)}\right)^{2}} \quad$ and $\sqrt{\sum_{i=N_{C}}^{N_{C}+N_{R}} \psi_{i j}\left(x^{a(j)}, y^{a(j)}, \theta^{a(j)}\right)^{2}}$, are, respectively, the spatial controllability of the first $N_{C}$ modes and the remaining residual modes. Regarding that the spatial controllability is the same as spatial $\mathscr{H}_{2}$ norm, for obtaining it, a limited number of modes are considered. The optimization criterion for locating actuators can be normalized as

$$
\begin{aligned}
J_{a}= & \frac{\sum_{j=1}^{N_{a}} \sqrt{\sum_{i=1}^{N_{C}} \psi_{i j}\left(x^{a(j)}, y^{a(j)}, \theta^{a(j)}\right)^{2}}}{\max \sum_{j=1}^{N_{a}} \sqrt{\sum_{i=1}^{N_{C}} \psi_{i j}\left(x^{a(j)}, y^{a(j)}, \theta^{a(j)}\right)^{2}}} \\
& -\lambda \frac{\sum_{j=1}^{N_{a}} \sqrt{\sum_{i=N_{C}}^{N_{C}+N_{R}} \psi_{i j}\left(x^{a(j)}, y^{a(j)}, \theta^{a(j)}\right)^{2}}}{\max \sum_{j=1}^{N_{a}} \sqrt{\sum_{i=N_{C}}^{N_{C}+N_{R}} \psi_{i j}\left(x^{a(j)}, y^{a(j)}, \theta^{a(j)}\right)^{2}}} .
\end{aligned}
$$

In (42), since the components of the numerator do not have the same numerical range, normalizing all of them to the same value, that is, the maximum value, is performed. If this normalization procedure would not have been taken into account, the resulting locations and orientations could not have been optimized correctly.

3.2. Optimal Placement of Sensors. For optimizing piezoelectric sensor locations, we rewrite (32) as

$$
\Phi^{\mathrm{se}}(t)=\mathbf{C}^{\prime} \dot{\mathbf{R}}=\mathbf{C} \dot{\mathbf{U}}=\sum_{i=1}^{n} C_{i} \dot{U}_{i},
$$

where $C_{i}$ is defined as 
TABLE 1: Terminology for GA.

\begin{tabular}{ll}
\hline Terminology & Description \\
\hline Fitness function & The function that should be optimized $(J$ in $(42)$ and $(47))$. \\
\hline Individual & $\begin{array}{l}\text { Any point that is utilized in the fitness function is an individual. The number of variables for finding } \\
\text { piezoelectric device positions and orientations is either } 3 \times N_{\text {se }} \text { or } 3 \times N_{a} \text { that is equal to the number of } \\
\text { individuals. }\end{array}$ \\
\hline Population & $\begin{array}{l}\text { An array of individuals forms the population. If the size of population is } N_{i} \text { and the number of variables or } \\
\text { individuals is } 3 \times N_{\text {se }} \text { or } 3 \times N_{a} \text {, then the population is shown by } N_{i} \text { by } 3 \times N_{\text {se }} \text { or } N_{i} \text { by } 3 \times N_{a} \text { matrices. }\end{array}$ \\
\hline Beginning & Creating a casual population is the first step of GA. \\
\hline Evaluation & The value of $J$ in $(42)$ and (47) to be evaluated for each population. \\
\hline Selection & Select two parents having the largest value of the fitness function. \\
\hline Crossover & Incorporate two parents that produce new children. \\
\hline Mutation & $\begin{array}{l}\text { In order that this operator produces the children, it applies a random change in parents. New children are } \\
\text { placed in new population }\end{array}$ \\
\hline
\end{tabular}

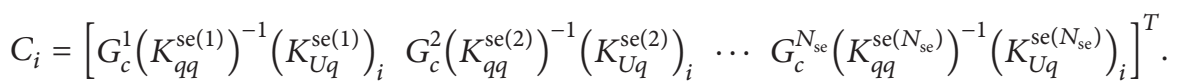

We find the optimal location of the $j$ th piezoelectric sensor by defining the spatial observability, function $\Theta_{i j}$, as

$$
\Theta_{i j}\left(x^{\mathrm{se}(j)}, y^{\mathrm{se}(j)}, \theta^{\mathrm{se}(j)}\right)=\left|C_{i}^{j}\right|=\left|G_{c}^{j}\left(K_{q q}^{\mathrm{se}(j)}\right)^{-1}\left(K_{U q}^{\mathrm{se}(j)}\right)_{i}\right|,
$$

where $\left(x^{\mathrm{se}}(j), y^{\mathrm{se}}(j)\right)$, and $\theta^{\mathrm{se}(j)}$ are the corner coordinates of the $j$ th sensor and its orientation, respectively. The orientation of sensors changes in the interval $(0 \leq \theta<\pi)$. In this work, the variables of sensor locations that should be optimized are as $\left\{\left(x^{\mathrm{se}(j)}, y^{\mathrm{se}(j)}, \theta^{\operatorname{se}(j)}\right)\right\}, j=1,2, \ldots, N_{\mathrm{se}}$.

The optimal placement of sensors can be found by maximizing the following criterion:

$$
\begin{aligned}
\sum_{j=1}^{N_{\mathrm{se}}} \sqrt{\sum_{i=1}^{N_{\mathrm{C}}} \Theta_{i j}\left(x^{\mathrm{se}(j)}, y^{\mathrm{se}(j)}, \theta^{\mathrm{se}(j)}\right)^{2}} \\
-\tilde{\lambda} \sum_{j=1}^{N_{\mathrm{se}}} \sqrt{\sum_{i=N_{C}}^{N_{C}+N_{R}} \Theta_{i j}\left(x^{\mathrm{se}(j)}, y^{\mathrm{se}(j)}, \theta^{\mathrm{se}(j)}\right)^{2}}
\end{aligned}
$$

which can be normalized as

$$
\begin{aligned}
J_{\mathrm{se}}= & \frac{\sum_{j=1}^{N_{\mathrm{se}}} \sqrt{\sum_{i=1}^{N_{C}} \Theta_{i j}\left(x^{\mathrm{se}(j)}, y^{\mathrm{se}(j)}, \theta^{\mathrm{se}(j)}\right)^{2}}}{\max \sum_{j=1}^{N_{\mathrm{se}}} \sqrt{\sum_{i=1}^{N_{C}} \Theta_{i j}\left(x^{\mathrm{se}(j)}, y^{\operatorname{se}(j)}, \theta^{\operatorname{se}(j)}\right)^{2}}} \\
& -\tilde{\lambda} \frac{\sum_{j=1}^{N_{\mathrm{se}}} \sqrt{\sum_{i=N_{C}}^{N_{C}+N_{R}} \Theta_{i j}\left(x^{\mathrm{se}(j)}, y^{\operatorname{se}(j)}, \theta^{\operatorname{se}(j)}\right)^{2}}}{\max \sum_{j=1}^{N_{\mathrm{se}}} \sqrt{\sum_{i=N_{C}}^{N_{C}+N_{R}} \Theta_{i j}\left(x^{\mathrm{se}(j)}, y^{\operatorname{se}(j)}, \theta^{\operatorname{se}(j)}\right)^{2}}} .
\end{aligned}
$$

By normalizing each Rayleigh-Ritz mode with respect to corresponding maximal energy that could be achieved from its corresponding mode, the criterion would search for the optimal configuration. In this criterion, the residual modes are considered and their influence on the optimization criterion is controlled by weighting constant, $\tilde{\lambda}$. By comparing (42) and (47), it is understood that, for optimizing both sensor and actuator locations, the same procedure should be implemented.

\section{Application of GA for Optimal Location of Piezoelectric Devices}

In this study, the GA is utilized for optimizing the piezoelectric device locations on the flexible plate. GA is a means by which the machine can simulate the mechanism of natural selection. This operation is done by searching in the design space for finding the best solution. The GA starts a search from a series of points, and, for performing the search procedure, it does not require the Jacobian of functions [33]. Many researchers for optimizing their work have used the GA [34-37]. The terminology used for GA is brought in Table 1.

Usually with the progress in the next generations, the algorithm converges to an asymptote point and the amount of fitness function improves. When the convergence is reached or the stopping criteria are satisfied, the search process stops. For optimization, a GA with the following configuration is considered in the work: population size: 200 , crossover rate: 0.8 , and number of generations: 50 .

Numerical simulations of the optimization process are analyzed in this section. We consider a simply supported rectangular plate for which the piezoelectric patches are attached to both upper and lower surfaces. The mechanical properties of aluminum that is used in this paper are $\rho=$ $2770 \mathrm{~kg} / \mathrm{m}^{3}, v=0.3, E=70 \mathrm{GPa}$, and $\zeta_{i}=0.0002$. We suppose that the piezoelectric sensors and actuators are perfectly bonded to the surfaces and they are made from the same 
TABLE 2: Geometrical properties of the plate and the piezoelectric sensors and actuators.

\begin{tabular}{lcc}
\hline & Plate & Piezoelectric patch \\
\hline$x$-length, $l_{x s}(\mathrm{~m})$ and $l_{x}(\mathrm{~m})$ & 1 & 0.08 \\
$y$-length, $l_{y s}(\mathrm{~m})$ and $l_{y}(\mathrm{~m})$ & 0.5 & 0.04 \\
Thickness, $h_{s t}$ and $h_{p}(\mathrm{~m})$ & 0.002 & 0.0001 \\
\hline
\end{tabular}

piezoelectric materials and symmetrically attached to the plate. Tables 2 and 3 give the geometrical and mechanical properties of materials that are used in this study.

By using the Rayleigh-Ritz method and expanding the transverse displacement of the plate in terms of a time dependent modal shape series, satisfying the geometric boundary conditions, we have:

$$
u_{3}(x, y, t)=\sum_{\kappa=1}^{k} \sum_{l=1}^{l} N_{u_{3}}^{\kappa l}(x, y) U^{\kappa l}(t)
$$

where $k$ and $l$ are the total number of mode shapes in the longitudinal $x$ and lateral $y$ directions, respectively. $\kappa$ and $\iota$ are the number of half-waves in the longitudinal $x$ and lateral $y$ directions; this series can be expressed as a matrix expansion:

$$
u_{3}=\left[\begin{array}{lllll}
N_{u_{3}}^{11} & N_{u_{3}}^{21} & N_{u_{3}}^{31} & \cdots & N_{u_{3}}^{k l}
\end{array}\right]\left[\begin{array}{lllll}
U^{11} & U^{21} & U^{31} & \cdots & U^{k l}
\end{array}\right]^{T} .
$$

We define

$$
\mathbf{N}_{\mathbf{u}_{3}}=\left[\begin{array}{lllll}
N_{u_{3}}^{11} & N_{u_{3}}^{21} & N_{u_{3}}^{31} & \cdots & N_{u_{3}}^{k l}
\end{array}\right] .
$$

The boundary conditions of plate are simply supported:

$$
\begin{gathered}
u_{1}=u_{2}=u_{3}=0 \quad \text { at } x=0, \\
x=l_{x s}, \quad y=0, \quad y=l_{y s},
\end{gathered}
$$

where $l_{x s}$ and $l_{y s}$ are the dimensions of rectangular plate.

For a simply supported plate, the modal shape functions that are used in this study are expanded in terms of double harmonic functions:

$$
N_{u_{3}}^{\kappa \iota}=\sin \left(\frac{\kappa \pi x}{l_{x s}}\right) \sin \left(\frac{\iota \pi y}{l_{y s}}\right),
$$

where $l_{x s}$ and $l_{y s}$ are the length and width of rectangular plate. For the purpose of vibration control, only the first five modes are considered that their natural frequencies are presented in Table 4. We have taken into account the influences of piezoelectric patches into our general formulation. However, we have neglected these effects in our solution for a simply supported rectangular plate, since the dimensions of piezoelectric patches are small in comparison with the host plate. We neglected their influences in calculating the natural frequencies and eigenmodes. Thus, in (18), $\mathbf{M}=\mathbf{M}^{\text {st }}$ and $\mathbf{K}_{\mathrm{UU}}=\mathbf{K}^{\text {st }}$. There are several examples considered here for showing the influence of GA that is used for locating the piezoelectric devices attached to a thin plate. At first, we discuss the values of $\lambda$ in the optimization procedure.
The different values of $\lambda$ that are substituted in (42) for the case of a single actuator and sensor are listed in Tables 5-7. In Table 5, the first mode is taken into account that would have the highest controllability, and else the next four modes are considered as the residual modes. As can be seen, when the values of $\lambda$ increase, the values of (40) decrease and the controllability decreases. It means that, for large values of $\lambda$, GA could not find the optimal position and orientation of the actuator. In Table 6, the second mode is taken into account that would have the highest controllability, and else the other modes are considered as the residual modes. As can be understood, similar to previous case, when the values of $\lambda$ increase, the values which appeared in (40) decrease and the controllability decreases, meaning that, for large values of $\lambda$, GA cannot find the optimal position and orientation of the actuator. In Table 7, the first two modes are unresidual modes, and else the other three modes are considered as the residual modes. For this case, several optimal positions are obtained that some of them are the nearly close for the different values of $\lambda$. Thus, the selection of $\lambda$ from disjoint ranges, as presented in Table 7, does not have any observable influence on finding the optimal positions and orientations. Thus, we would choose the value of $\lambda$ equal to two.

In the first two simulations, the first two lowest modes would seem to have the highest controllability and observability and else the remaining three modes are considered as the residual modes. Because the fitness functions for finding sensor and actuator (transducer) locations and orientations are the same, herein, we only discuss how to find the actuator locations and orientations. In Figure 2(a), the best and mean values of the fitness function for finding the optimal location of one transducer attached to the plate are shown. As can be seen, the best value for the objective function is equal to 1.68. The optimal corner point coordinates for a single-transducer are $(0.657 \mathrm{~m}, 0.206 \mathrm{~m})$, while the transducer orientation in this point is $0.9 \mathrm{rad}$. The best and mean fitness function values for finding the optimal locations of two transducers are indicated in Figure 2(b). In the first generations, the best value of fitness function is improved rapidly that is because of being too far away from the optimal point. In the other simulations of this section, the first three lower modes are controlled and the remaining two modes are considered as residual modes. The evolution of the best and mean values of the fineness function is presented in Figure 3. The optimal positions of these simulations are listed in Tables 8 and 9.

\section{Results and Discussion}

5.1. Controller Design. For active vibration control, a negative velocity feedback control algorithm is used. This feedback strategy increases the depreciation rate; thus it is an effective way for reducing the oscillatory amplitude. A simple algorithm of this type is used in such a way that the actuator voltage can be obtained with an amplifier gain and a change of polarity on the sensor voltage as follows:

$$
\mathbf{v}(t)^{\mathbf{a}}=-\mathbf{G}_{\mathbf{C o}} \Phi^{\mathbf{s e}}(t),
$$

where $\mathbf{G}_{\mathbf{C o}}$ is a matrix of gains of the amplifier that is used in the feedback control. 


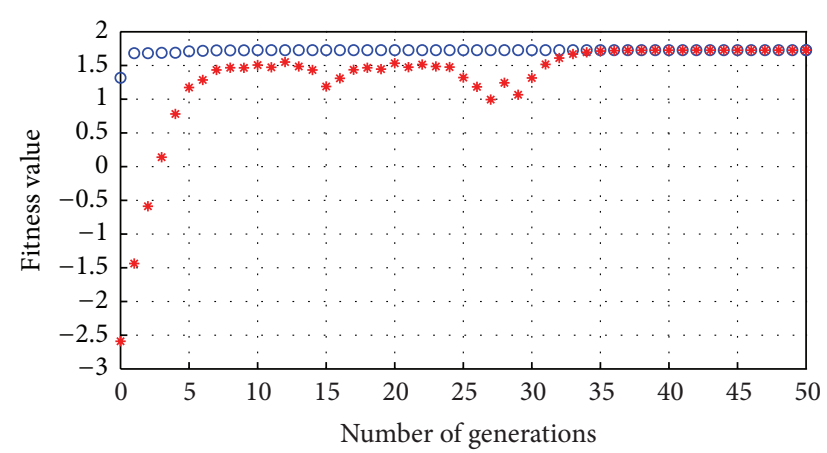

- Best value

* Mean value

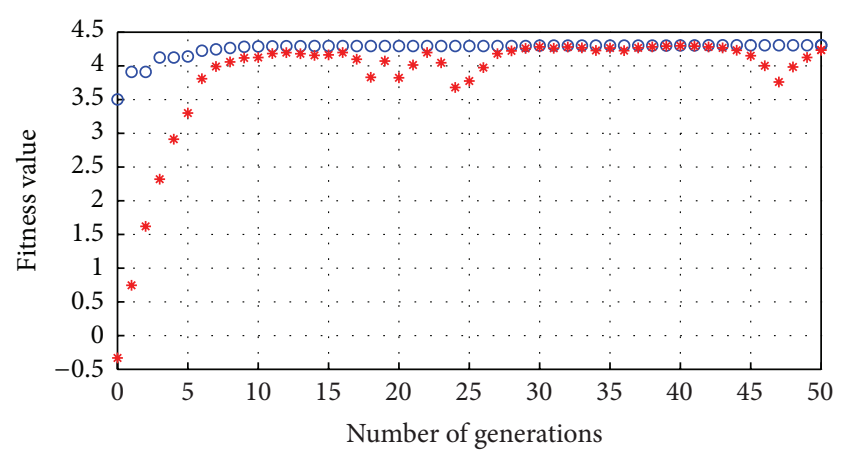

- Best value

* Mean value

(a)

(b)

Figure 2: Evolution of the mean and best values of fitness function for finding actuator-sensor locations when the first two modes are controlled, while the next three modes are the residual modes: (a) one actuator-sensor and (b) a pair of actuator-sensors.

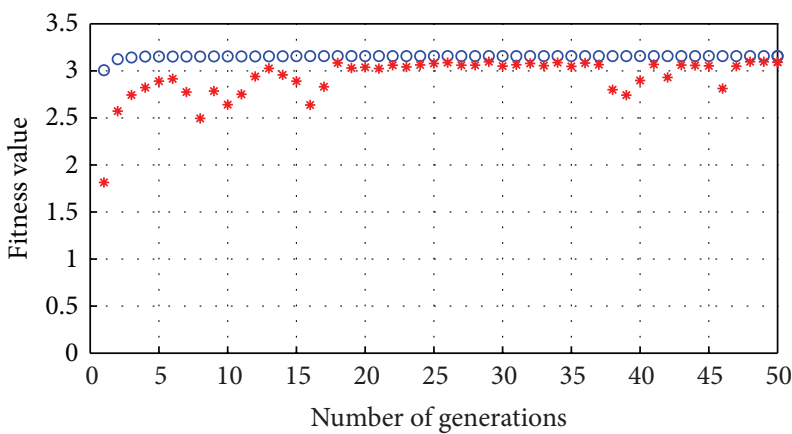

- Best value

* Mean value

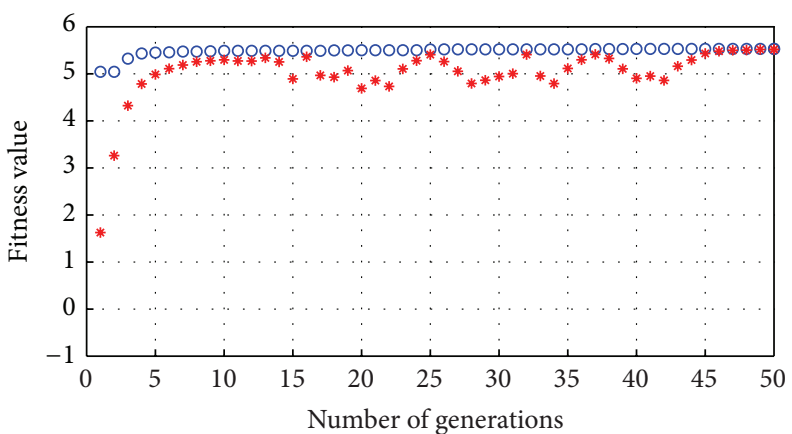

- Best value

* Mean value

(a)

(b)

FIGURE 3: Evolution of the mean and best values of fitness function for finding actuator-sensor locations when the first three modes are controlled, while the next two modes are the residual modes: (a) one actuator-sensor and (b) a pair of actuator-sensors.

In next subsection, several simulations are presented for illustrating the influence of optimization procedure and active control algorithm.

5.2. Simulation 1. In the first simulation, the first two lower modes should be controlled and the remaining three modes are considered as residual modes. The transducers should be located at random and optimal positions that are listed in Table 8. For exciting a larger number of system mode shapes, we have applied the step function concentrated load at a location off-center of plate. The frequency responses of the plate, when excited by a concentrated force and located at $x=0.43 \mathrm{~m}$ and $y=0.21 \mathrm{~m}$ for different number of sensors and actuators, are plotted in Figure 4. As can be seen, the optimization procedure is more effective for the amplitude vibration reduction as contributed to arbitrary location of actuators/sensors. By locating the actuators and sensors in the optimal locations, more damping is developed in the plate. When the sensors are located at random locations, we could not observe the first two modes in the response, and when the actuators are positioned at random locations the maximum mechanical energy cannot be transmitted to the first two modes. However if the sensors are located at the optimal locations, the contributed modes could be observed. Furthermore, if the actuators are located at their optimal positions, the maximum mechanical energy could be transmitted to the contributed modes, causing the amplitude of vibration could be reduced more. The values of spatial controllability (40) are shown in Tables 10 and 11. The transducers are located in position of Table 6 . The first two modes are the controlled modes and the modes 3-5 are the residual modes. By comparing the values of these two tables, it can be seen that, for the optimal locations, the values of the system controllability for the first two modes are greater than the values of an arbitrarily selected location. Thus, the optimization procedure would seem to be effective. In Figure 5, two actuators are used for active vibration control of the simply supported rectangular plate under consideration. In this figure, we would compare the optimal configurations in Table 8 and use the optimal locations of one actuator 


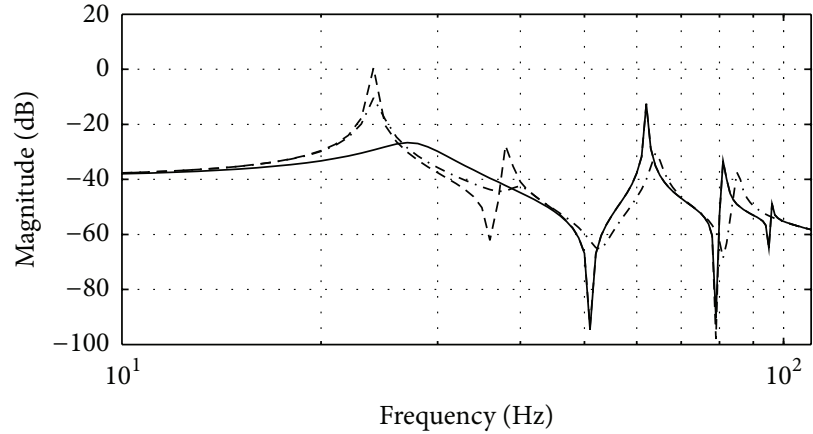

$\begin{array}{lll}\text { - Optimal location } & \text { - - - Without control } \\ \text {-.- Arbitrarily located } & \end{array}$

(a)

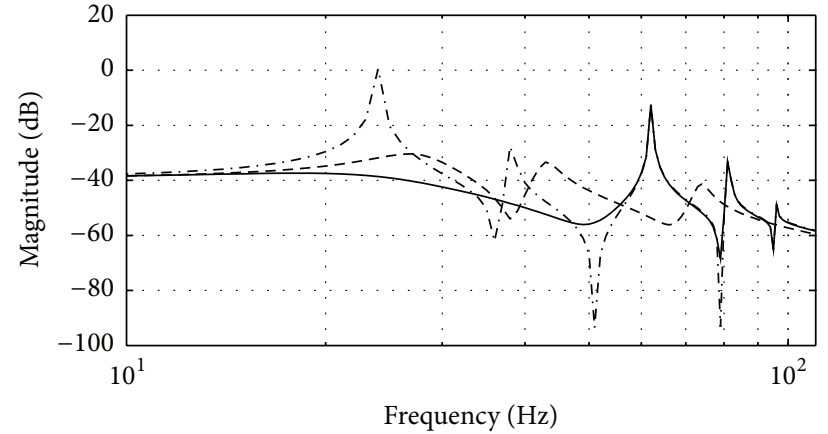

-..- Without control - Arbitrarily located

(b)

FIGURE 4: Frequency response of the plate controlled by the piezoelectric actuator-sensor patches when the first two modes are controlled, while the next three modes are the residual modes: (a) one actuator-sensor and (b) a pair of actuator-sensors.

TABLE 3: Material properties of piezoelectric transducers made of PZT-5H from [28].

\begin{tabular}{|c|c|c|c|}
\hline Mechanical properties & $c_{11}^{D}=c_{22}^{D}=131.6 \mathrm{GPa}$ & $\begin{array}{cc}c_{12}^{D}=84.2 \mathrm{GPa} & c_{66}^{D}=3 \mathrm{GPa}\end{array}$ & $\rho=7800 \mathrm{~kg} / \mathrm{m}^{3}$ \\
\hline Electrical property & & $\beta_{33}^{s}=1.48 \times 10^{8} \mathrm{~m} / \mathrm{F}$ & \\
\hline Coupling coefficients & & $h_{13}=h_{23}=-2.72 \times 10^{9} \mathrm{~N} / \mathrm{C}$ & \\
\hline
\end{tabular}

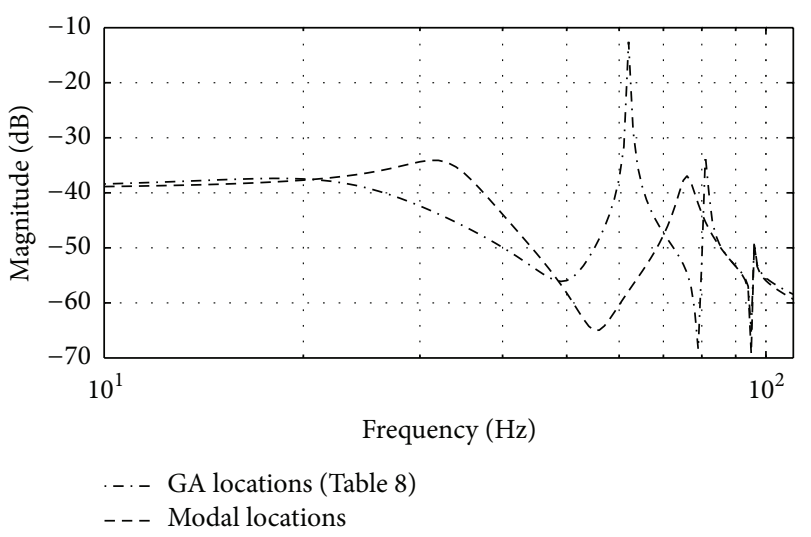

FIGURE 5: Comparison of the optimal configurations in Table 8 and use of the optimal locations of one actuator activated for the first mode and the other actuator for the second mode.

activated for the first mode and the other actuator for the second mode. As can be seen, the results based on mathematical procedure used based on GA and represented in Table 8 are more effective than the individual activations of each actuator for each mode.

5.3. Simulation 2. In the second simulation, the first three lower modes must be controlled and the remaining two modes are residual modes. The transducers should be located at random and optimal positions that are listed in Table 9. The frequency responses of the plate, excited by a concentrated force and located at $x=0.43 \mathrm{~m}$ and $y=0.21 \mathrm{~m}$, for single and double transducer configurations, are presented in Figure 6. As can be seen, the optimization criteria are effective in having more damping effect. In Figure 7, the influences of the
TABLE 4: Natural frequencies of the plate (before active control).

\begin{tabular}{lccccc}
\hline Mode number & 1 & 2 & 3 & 4 & 5 \\
\hline $\begin{array}{l}\text { Longitudinal and } \\
\text { circumferential modes }(\kappa, \iota)\end{array}$ & $(1,1)$ & $(2,1)$ & $(3,1)$ & $(1,2)$ & $(2,2)$ \\
\hline Natural frequencies $[\mathrm{Hz}]$ & 23.88 & 38.22 & 62.11 & 81.23 & 95.57 \\
\hline
\end{tabular}

number of sensors and actuators are compared in the control of vibration. In this simulation, the sensors and actuators are located in the optimal positions and the first two lower modes must be controlled. It can be concluded that when a pair of actuators and sensors are used the damping effect increases and the amplitude vibration of plate reduces more.

\section{Conclusions}

In the present study, the active vibration control and optimal position of piezoelectric patches attached to a thin plate were analyzed. For deriving the equation of motion and sensor output equation, Hamilton's principle and Rayleigh-Ritz method were used. In the next step, the optimal positions and orientations of piezoelectric actuators and sensors attached to a rectangular plate were determined based on the concept of spatial controllability/observability and considering residual modes, for reducing the spillover effect. GA was utilized for optimizing the locations and orientations of piezoelectric devices. The results indicated that, by locating piezoelectric sensors and actuators in the optimal positions, the damping effect could increase and the amplitudes of plate vibration were reduced more effectively. Furthermore, in general, the active vibration control was effective in the vibration control of the thin plate. 
TABLE 5: The effect of values of the weight coefficients on the controllability for a single actuator for the case that only the first mode is controlled.

\begin{tabular}{|c|c|c|c|c|c|c|c|c|}
\hline \multirow{2}{*}{$\begin{array}{l}\text { Actuator longitudinal } \\
\text { coordinate } x^{a}\end{array}$} & \multirow{2}{*}{ Actuator lateral coordinate $y^{a}$} & \multirow{2}{*}{ Actuator orientation $\theta^{a}$} & \multirow{2}{*}{ Weight coefficient $\lambda$} & \multicolumn{5}{|c|}{ Values of controllability of $i$ th mode } \\
\hline & & & & 1 & 2 & 3 & 4 & 5 \\
\hline $0.487 \mathrm{~m}$ & $0.207 \mathrm{~m}$ & $0.818 \mathrm{rad}$ & 0.500 & 0.022 & 0.000 & 0.057 & 0.000 & 0.000 \\
\hline $0.538 \mathrm{~m}$ & $0.226 \mathrm{~m}$ & $2.114 \mathrm{rad}$ & 0.100 & 0.022 & 0.000 & 0.056 & 0.000 & 0.000 \\
\hline $0.494 \mathrm{~m}$ & $0.455 \mathrm{~m}$ & $1.188 \mathrm{rad}$ & 1.000 & 0.000 & 0.000 & 0.000 & 0.000 & 0.000 \\
\hline $0.469 \mathrm{~m}$ & $0.457 \mathrm{~m}$ & $1.350 \mathrm{rad}$ & 2.000 & 0.000 & 0.000 & 0.000 & 0.000 & 0.000 \\
\hline $0.501 \mathrm{~m}$ & $0.455 \mathrm{~m}$ & $1.121 \mathrm{rad}$ & 3.000 & 0.000 & 0.000 & 0.000 & 0.000 & 0.000 \\
\hline
\end{tabular}

TABLE 6: The effect of values of the weight coefficients on the controllability for a single actuator for the case that only the second mode is controlled.

\begin{tabular}{|c|c|c|c|c|c|c|c|c|}
\hline \multirow{2}{*}{$\begin{array}{l}\text { Actuator longitudinal } \\
\text { coordinate } x^{a}\end{array}$} & \multirow{2}{*}{ Actuator lateral coordinate $y^{a}$} & \multirow{2}{*}{ Actuator orientation $\theta^{a}$} & \multirow{2}{*}{ Weight coefficient $\lambda$} & \multicolumn{5}{|c|}{ Values of controllability of $i$ th mode } \\
\hline & & & & 1 & 2 & 3 & 4 & 5 \\
\hline $0.294 \mathrm{~m}$ & $0.217 \mathrm{~m}$ & $1.856 \mathrm{rad}$ & 0.100 & 0.012 & 0.035 & 0.021 & 0.000 & 0.000 \\
\hline $0.677 \mathrm{~m}$ & $0.206 \mathrm{~m}$ & $1.329 \mathrm{rad}$ & 0.500 & 0.016 & 0.026 & 0.000 & 0.000 & 0.000 \\
\hline $0.489 \mathrm{~m}$ & $0.455 \mathrm{~m}$ & $1.104 \mathrm{rad}$ & 1.000 & 0.000 & 0.000 & 0.000 & 0.000 & 0.000 \\
\hline $0.878 \mathrm{~m}$ & $0.459 \mathrm{~m}$ & $0.705 \mathrm{rad}$ & 2.000 & 0.000 & 0.000 & 0.000 & 0.000 & 0.000 \\
\hline $0.503 \mathrm{~m}$ & $0.455 \mathrm{~m}$ & $1.167 \mathrm{rad}$ & 3.000 & 0.000 & 0.000 & 0.000 & 0.000 & 0.000 \\
\hline
\end{tabular}

TABLE 7: The effect of values of the weight coefficients on the controllability for a single actuator for the case that the first two modes are controlled.

\begin{tabular}{|c|c|c|c|c|c|c|c|c|}
\hline \multirow{2}{*}{$\begin{array}{l}\text { Actuator longitudinal } \\
\text { coordinate } x^{a}\end{array}$} & \multirow{2}{*}{ Actuator lateral coordinate $y^{a}$} & \multirow{2}{*}{ Actuator orientation $\theta^{a}$} & \multirow{2}{*}{ Weight coefficient $\lambda$} & \multicolumn{5}{|c|}{ Values of controllability of $i$ th mode } \\
\hline & & & & 1 & 2 & 3 & 4 & 5 \\
\hline $0.363 \mathrm{~m}$ & $0.250 \mathrm{~m}$ & $2.672 \mathrm{rad}$ & 0.100 & 0.016 & 0.029 & 0.001 & 0.000 & 0.000 \\
\hline $0.670 \mathrm{~m}$ & $0.205 \mathrm{~m}$ & $1.178 \mathrm{rad}$ & 0.500 & 0.016 & 0.026 & 0.000 & 0.000 & 0.000 \\
\hline $0.314 \mathrm{~m}$ & $0.210 \mathrm{~m}$ & $0.651 \mathrm{rad}$ & 2.000 & 0.016 & 0.026 & 0.000 & 0.000 & 0.000 \\
\hline $0.695 \mathrm{~m}$ & $0.215 \mathrm{~m}$ & $1.789 \mathrm{rad}$ & 3.000 & 0.017 & 0.026 & 0.000 & 0.000 & 0.000 \\
\hline $0.702 \mathrm{~m}$ & $0.223 \mathrm{~m}$ & $2.021 \mathrm{rad}$ & 5.000 & 0.017 & 0.026 & 0.000 & 0.000 & 0.000 \\
\hline
\end{tabular}

TABLE 8: GA-optimal and random configurations of piezoelectric transducers attached to the rectangular plate. The first two modes are the dominant modes, while the next three modes are the residual modes.

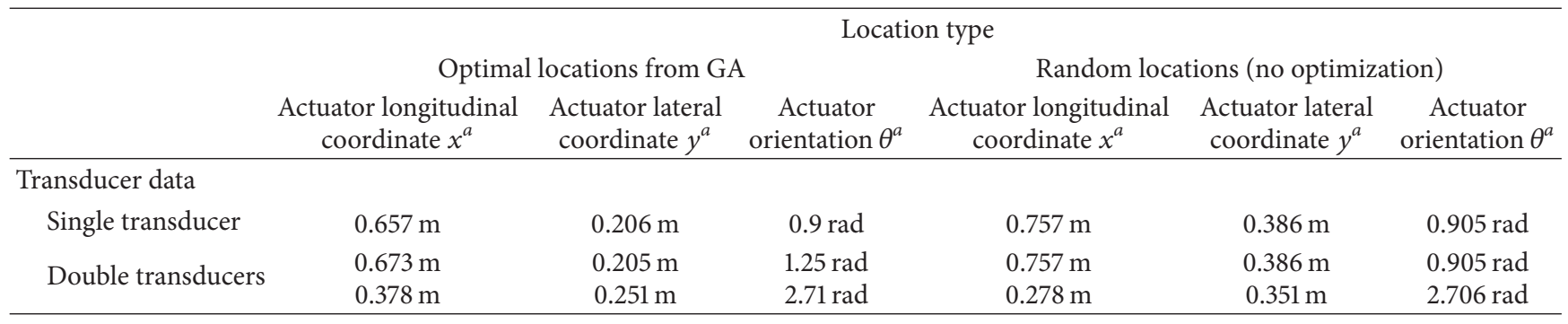

TABLE 9: GA-optimal and random configurations of piezoelectric transducers attached to the rectangular plate. The first three modes are the dominant modes, while the next two modes are the residual modes.

\begin{tabular}{|c|c|c|c|c|c|c|}
\hline & \multicolumn{6}{|c|}{ Location type } \\
\hline & \multicolumn{3}{|c|}{ Optimal locations from GA } & \multicolumn{3}{|c|}{ Random locations (no optimization) } \\
\hline & $\begin{array}{l}\text { Actuator longitudinal } \\
\text { coordinate } x^{a}\end{array}$ & $\begin{array}{l}\text { Actuator lateral } \\
\text { coordinate } y^{a}\end{array}$ & $\begin{array}{c}\text { Actuator } \\
\text { orientation } \theta^{a}\end{array}$ & $\begin{array}{l}\text { Actuator longitudinal } \\
\text { coordinate } x^{a}\end{array}$ & $\begin{array}{l}\text { Actuator lateral } \\
\text { coordinate } y^{a}\end{array}$ & $\begin{array}{c}\text { Actuator } \\
\text { orientation } \theta^{a}\end{array}$ \\
\hline \multicolumn{7}{|l|}{ Transducer data } \\
\hline Single transducer & $0.481 \mathrm{~m}$ & $0.227 \mathrm{~m}$ & $2.14 \mathrm{rad}$ & $0.531 \mathrm{~m}$ & $0.257 \mathrm{~m}$ & $2.14 \mathrm{rad}$ \\
\hline Double transducers & $\begin{array}{l}0.437 \mathrm{~m} \\
0.475 \mathrm{~m}\end{array}$ & $\begin{array}{l}0.215 \mathrm{~m} \\
0.152 \mathrm{~m}\end{array}$ & $\begin{array}{c}1.8 \mathrm{rad} \\
1.49 \mathrm{rad}\end{array}$ & $\begin{array}{l}0.337 \mathrm{~m} \\
0.275 \mathrm{~m}\end{array}$ & $\begin{array}{l}0.115 \mathrm{~m} \\
0.352 \mathrm{~m}\end{array}$ & $\begin{array}{l}1.796 \mathrm{rad} \\
1.494 \mathrm{rad}\end{array}$ \\
\hline
\end{tabular}




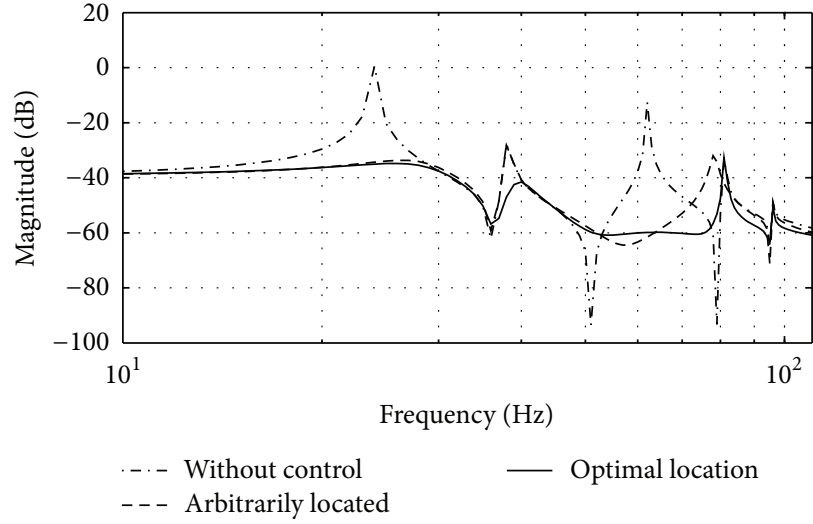

(a)

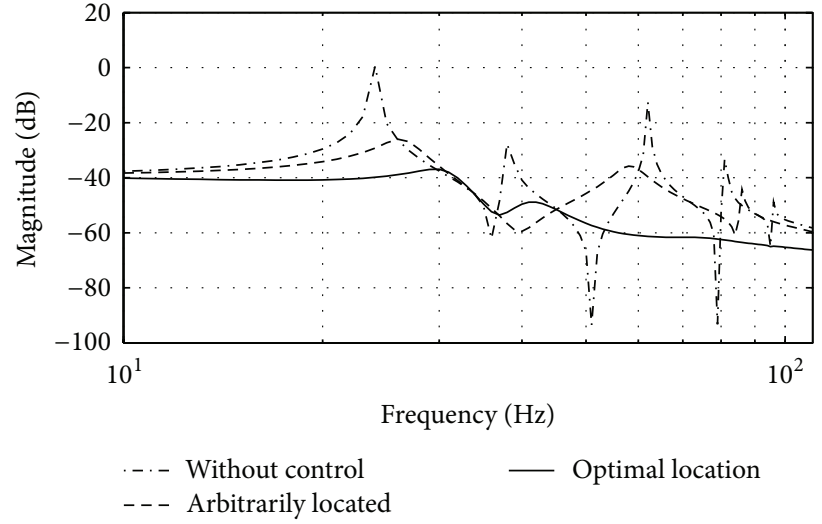

(b)

FigurE 6: Frequency response of the plate controlled by the piezoelectric actuator-sensor. The first three modes are the controlled modes, while the next two modes are the residual modes: (a) one actuator-sensor and (b) two actuator-sensors.

TABLE 10: The effect of values of controllability for optimal location, whenever the first two modes are controlled.

\begin{tabular}{lccccc}
\hline & \multicolumn{5}{c}{ Values of controllability of $i$ th mode } \\
\hline Number of actuators & 1 & 2 & 3 & 4 & 5 \\
One actuator & 0.033 & 0.053 & 0.000 & 0.000 & 0.000 \\
Two actuators & 0.016 & 0.026 & 0.000 & 0.000 & 0.000 \\
\hline
\end{tabular}

TABLE 11: The effect of values of controllability for random location, whenever the first two modes are controlled.

\begin{tabular}{lccccc}
\hline & \multicolumn{5}{c}{ Values of controllability of $i$ th mode } \\
\hline Number of actuators & 1 & 2 & 3 & 4 & 5 \\
One actuator & 0.001 & 0.004 & 0.010 & 0.008 & 0.032 \\
Two actuators & 0.001 & 0.007 & 0.018 & 0.010 & 0.040 \\
\hline
\end{tabular}

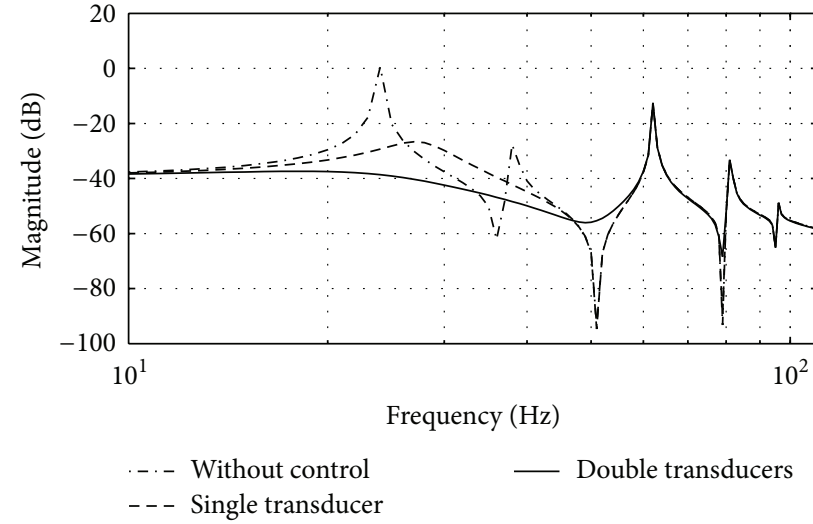

FIGURE 7: Comparison of frequency response of the plate controlled by different number of piezoelectric actuator-sensors.

\section{Nomenclature}

A: $\quad$ The area of piezoelectric patches

$\mathbf{B}_{\mathbf{U}}^{\mathbf{m}}, \mathbf{B}_{\mathbf{U}}^{\mathbf{m}}$ : The strain-displacement transformation matrices

$\mathrm{c}_{\mathrm{st}}$ : The matrix of elastic constants of structure

$c^{D}$ : The elasticity matrix of piezoelectric patches
D:

f:

$G(x, y, s):$

$\|G\|_{2}^{2}$

$\mathrm{G}_{\mathrm{c}}$ :

$\mathrm{G}_{\mathrm{Co}}$ :

h:

H:

ise: $^{\text {se }}$

$J_{a}, J_{\mathrm{se}}$ :

K:

$k, l: \quad \quad$ The total number of mode shapes in the

longitudinal $x$ and lateral $y$ directions

The kinetic and potential energy of system

$\mathrm{K}_{\mathrm{UU}}$ : $\quad$ The passive stiffness matrix of the system (structure/piezopatches)

$\mathbf{K}^{\text {st }}, \mathbf{K}_{\mathbf{D}}^{\text {se }}$, and $\mathbf{K}_{\mathbf{D}}^{\mathbf{a}}$ : The stiffness matrices of the structure, piezoelectric sensors, and actuators 


$$
\begin{aligned}
& \mathbf{K}_{\mathbf{U q}}^{\mathbf{s e}}, \mathbf{K}_{\mathbf{U q}}^{\mathbf{a}}: \\
& \mathbf{K}_{\mathbf{q q}}^{\mathbf{s e}}, \mathbf{K}_{\mathbf{q q}}^{\mathbf{a}}: \\
& l_{x s}, l_{y s}:
\end{aligned}
$$

M: $\mathbf{M}^{\text {st }}, \mathbf{M}^{\text {se }}$, and $\mathbf{M}^{\mathbf{a}}$ :

$n:$

$\mathbf{N}_{\mathbf{u}_{1}}, \mathbf{N}_{\mathbf{u}_{2}}$, and $\mathbf{N}_{\mathbf{u}_{3}}$ :

$N_{C}$ :

$N_{R}$ :

$N_{a}, N_{\mathrm{se}}:$

q:

R:

$\mathbf{R}_{S}^{\mathbf{j}}, \mathbf{R}_{\mathbf{D}}^{\mathbf{j}}$ :

$S:$

$S_{x x}, S_{y y}$, and $S_{x y}$ :

$\mathbf{U}_{1}, \mathbf{U}_{2}$, and $\mathbf{U}_{3}$ :

$u_{1}, u_{2}$, and $u_{3}$ :

$\dot{u}_{1}, \dot{u}_{2}$, and $\dot{u}_{3}$ :

v:

$V^{\text {st }}, V^{a}$, and $V^{\text {se }}$ :

$\beta_{\mathrm{s}}$ :

$\Phi^{\text {se }}$ :

$\kappa, l$ :

$\tilde{\lambda}, \lambda:$

$\Lambda_{\mathbf{n}}$ :

$\Lambda_{n}^{2}$ :

$\rho^{\text {st }}, \rho^{a}$, and $\rho^{\text {se }}$ :

$\Upsilon_{\mathrm{a}}$ :

\section{Conflict of Interests}

The authors declare that there is no conflict of interests regarding the publication of this paper.

\section{References}

[1] H. Boudaoud, S. Belouettar, E. M. Daya, and M. Potier-Ferry, "A shell finite element for active-passive vibration control of composite structures with piezoelectric and viscoelastic layers,"
Mechanics of Advanced Materials and Structures, vol. 15, no. 3-4, pp. 208-219, 2008.

[2] A. Ajit, K. K. Ang, and C. M. Wang, "Shape control of statically indeterminate laminated beams with piezoelectric actuators," Mechanics of Advanced Materials and Structures, vol. 10, no. 2, pp. 145-160, 2003.

[3] R. Kumar, B. K. Mishra, and S. C. Jain, "Static and dynamic analysis of smart cylindrical shell," Finite Elements in Analysis and Design, vol. 45, no. 1, pp. 13-24, 2008.

[4] M. K. Kwak and S. Heo, "Active vibration control of smart grid structure by multiinput and multioutput positive position feedback controller," Journal of Sound and Vibration, vol. 304, no. 1-2, pp. 230-245, 2007.

[5] P. Cupial, "Calculation of the optimum controls of transient vibrations of smart beams and plates," Mechanics of Advanced Materials and Structures, vol. 15, no. 3-4, pp. 258-268, 2008.

[6] M. Yiqi and F. Yiming, "Nonlinear dynamic response and active vibration control for piezoelectric functionally graded plate," Journal of Sound and Vibration, vol. 329, no. 11, pp. 2015-2028, 2010.

[7] X. Q. He, T. Y. Ng, S. Sivashanker, and K. M. Liew, "Active control of FGM plates with integrated piezoelectric sensors and actuators," International Journal of Solids and Structures, vol. 38, no. 9, pp. 1641-1655, 2001.

[8] R. R. Orszulik and J. Shan, "Active vibration control using genetic algorithm-based system identification and positive position feedback," Smart Materials and Structures, vol. 21, no. 5, Article ID 055002, 2012.

[9] S. Julai and M. O. Tokhi, "Vibration suppression of flexible plate structures using swarm and genetic optimization techniques," Journal of Low Frequency Noise Vibration and Active Control, vol. 29, no. 4, pp. 293-318, 2010.

[10] M. D. Kulkarni, G. Kumar, P. M. Mujumdar, and A. Joshi, "Active control of vibration modes of a wing box by piezoelectric stack actuators," in Proceedings of the 51st AIAA/ASME/ASCE/ AHS/ASC Structures, Structural Dynamics and Materials Conference, Orlando, Fla, USA, April 2010.

[11] Y. H. Zhao and H. Y. Hu, "Active control of vertical tail buffeting by piezoelectric actuators," Journal of Aircraft, vol. 46, no. 4, pp. 1167-1175, 2009.

[12] K. R. Kumar and S. Narayanan, "Active vibration control of beams with optimal placement of piezoelectric sensor/actuator pairs," Smart Materials and Structures, vol. 17, no. 5, Article ID 055008, 2008.

[13] I. Bruant, G. Coffignal, F. Lene, and M. Verge, "A methodology for determination of piezoelectric actuator and sensor location on beam structures," Journal of Sound and Vibration, vol. 243, no. 5, pp. 861-882, 2001.

[14] A. Hać and L. Liu, "Sensor and actuator location in motion control of flexible structures," Journal of Sound and Vibration, vol. 167, no. 2, pp. 239-261, 1993.

[15] I. Bruant, F. Pablo, and O. Polit, "Active control of laminated plates using a piezoelectric finite element," Mechanics of Advanced Materials and Structures, vol. 15, no. 3-4, pp. 276-290, 2008.

[16] D. Halim and O. R. Moheimani, "An optimization approach to optimal placement of collocated piezoelectric actuators and sensors on a thin plate," Mechatronics, vol. 13, no. 1, pp. 27-47, 2003.

[17] S. M. Yang and Y. J. Lee, "Optimization of noncollocated sensor/actuator location and feedback gain in control systems," 
Smart Materials and Structures, vol. 2, no. 2, article 005, pp. 96102, 1993.

[18] A. A. Rader, F. F. Afagh, A. Yousefi-Koma, and D. G. Zimcik, "Optimization of piezoelectric actuator configuration on a flexible fin for vibration control using genetic algorithms," Journal of Intelligent Material Systems and Structures, vol. 18, no. 10, pp. 1015-1033, 2007.

[19] P. Ambrosio, F. Resta, and F. Ripamonti, "An $\mathrm{H}_{2}$ norm approach for the actuator and sensor placement in vibration control of a smart structure," Smart Materials and Structures, vol. 21, no. 12, Article ID 125016, 2012.

[20] M. R. Sajizadeh and I. Z. M. Darus, "Optimal location of sensor for active vibration control of flexible square plate," in Proceedings of the 10th International Conference on Information Sciences, Signal Processing and their Applications (ISSPA '10), pp. 393-396, Kuala Lumpur, Malaysia, May 2010.

[21] F. Qian, J. Wang, and L. Qu, "Optimal placements of piezoelectric patch using genetic algorithm in structure vibration control," Acta Mechanica Solida Sinica, vol. 32, no. 4, pp. 398404, 2011.

[22] I. Bruant, L. Gallimard, and S. Nikoukar, "Optimization of piezoelectric sensors location and number using a genetic algorithm," Mechanics of Advanced Materials and Structures, vol. 18, no. 7, pp. 469-475, 2011.

[23] Z.-C. Qiu, X.-M. Zhang, H.-X. Wu, and H.-H. Zhang, "Optimal placement and active vibration control for piezoelectric smart flexible cantilever plate," Journal of Sound and Vibration, vol. 301, no. 3-5, pp. 521-543, 2007.

[24] A. M. Sadri, J. R. Wright, and R. J. Wynne, "Modelling and optimal placement of piezoelectric actuators in isotropic plates using genetic algorithms," Smart Materials and Structures, vol. 8, no. 4, pp. 490-498, 1999.

[25] I. Bruant, L. Gallimard, and S. Nikoukar, "Optimal piezoelectric actuator and sensor location for active vibration control, using genetic algorithm," Journal of Sound and Vibration, vol. 329, no. 10, pp. 1615-1635, 2010.

[26] J.-H. Han and I. Lee, "Optimal placement of piezoelectric sensors and actuators for vibration control of a composite plate using genetic algorithms," Smart Materials and Structures, vol. 8, no. 2, pp. 257-267, 1999.

[27] I. Bruant and L. Proslier, "Optimal location of actuators and sensors in active vibration control," Journal of Intelligent Material Systems and Structures, vol. 16, no. 3, pp. 197-206, 2005.

[28] D. J. Leo, Engineering Analysis of Smart Material Systems, John Wiley and Sons, New York, NY, USA, 2007.

[29] D. J. Inman, Vibration with Control John, Wiley and Sons, New York, NY, USA, 2006.

[30] S. O. Reza Moheimani, D. Halim, and A. J. Fleming, Spatial Control of Vibration Theory and Experiments, World Scientific Publishing, New York, NY, USA, 2002.

[31] S. O. R. Moheimani and M. Fu, "Spatial H2 norm of flexible structures and its application in model order selection," in Proceedings of the 37th IEEE Conference on Decision and Control (CDC '98), pp. 3623-3624, Tampa, Fla, USA, December 1998.

[32] S. O. R. Moheimani and T. Ryall, "Considerations on placement of piezoceramic actuators that are used in structural vibration control," in Proceedings of the 38th IEEE Conference on Decision and Control (CDC '99), pp. 1118-1123, Phoenix, Ariz, USA, December 1999.

[33] S. N. Sivanandam and S. N. Deepa, Introduction to Genetic Algorithms, Springer, New York, NY, USA, 2008.
[34] M. Biglar, H. R. Mirdamadi, and M. Danesh, "Optimal locations and orientations of piezoelectric transducers on cylindrical shell based on gramians of contributed and undesired RayleighRitz modes using genetic algorithm," Journal of Sound and Vibration, vol. 333, no. 5, pp. 1224-1244, 2014.

[35] L. Costa, P. Oliveira, I. N. Figueiredo, and R. Leal, "Actuator effect of a piezoelectric anisotropic plate model," Mechanics of Advanced Materials and Structures, vol. 13, no. 5, pp. 403-417, 2006.

[36] D. Marinova, "Finite element formulation for analysis and shape regulating of plates with laminated piezoelectric material," Mechanics of Advanced Materials and Structures, vol. 16, no. 3, pp. 224-235, 2009.

[37] P. Vannucci, "A new general approach for optimizing the performances of smart laminates," Mechanics of Advanced Materials and Structures, vol. 18, no. 7, pp. 548-558, 2011. 

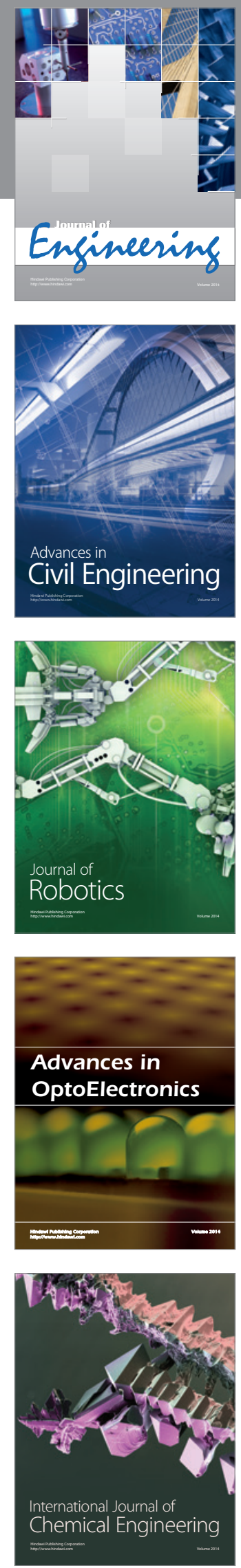

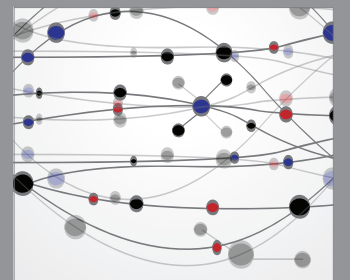

The Scientific World Journal
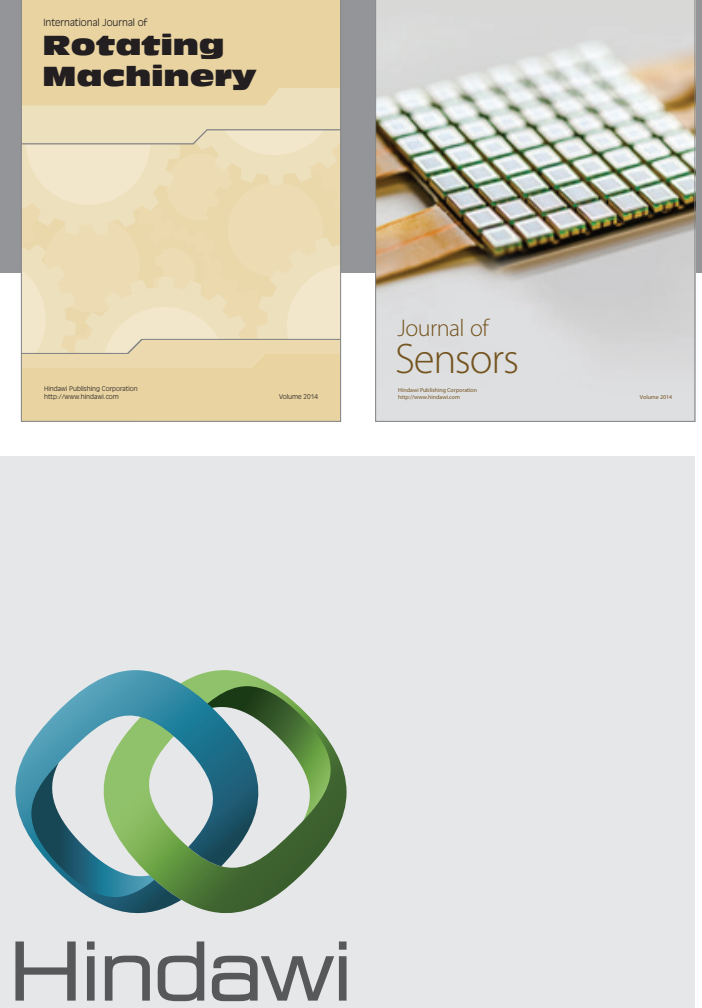

Submit your manuscripts at http://www.hindawi.com
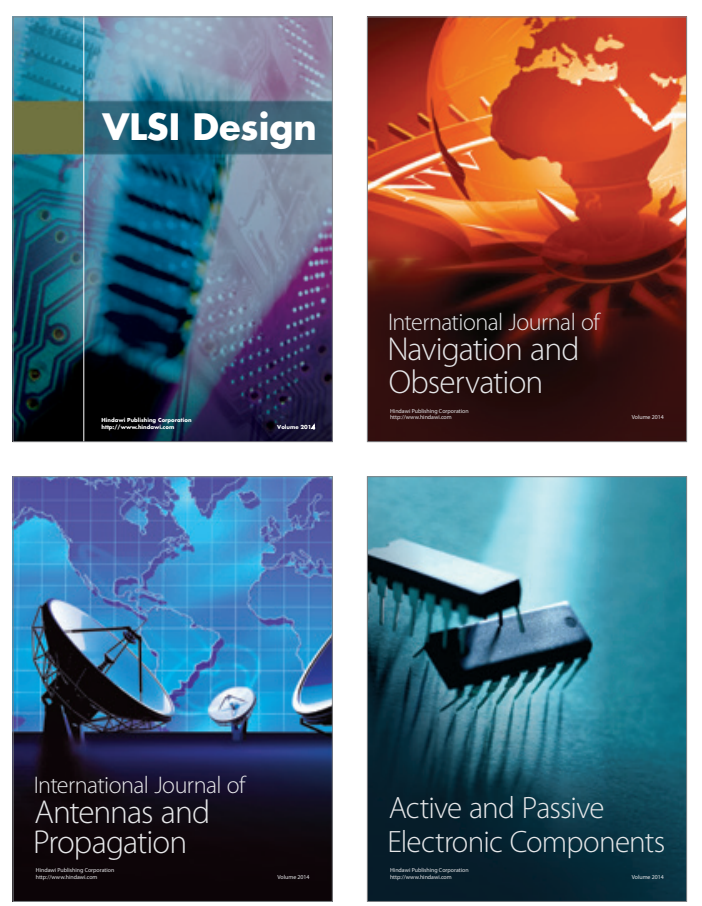
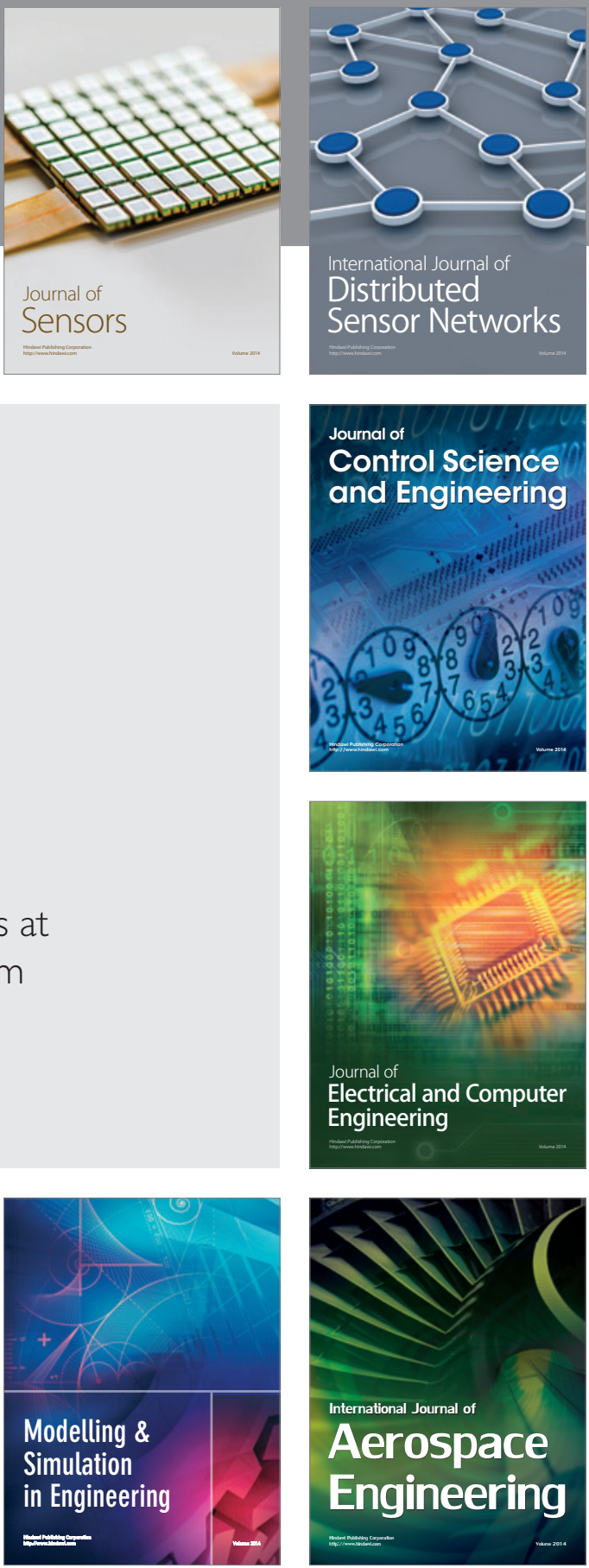

Journal of

Control Science

and Engineering
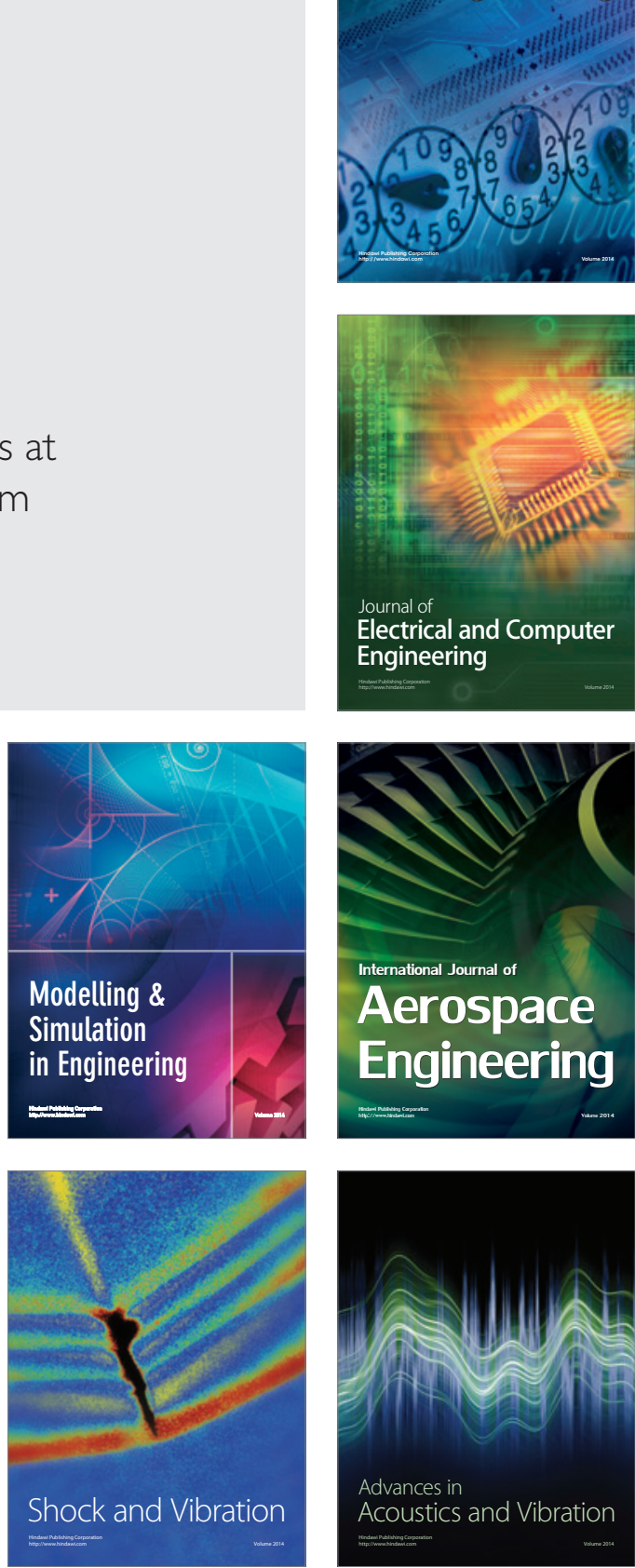